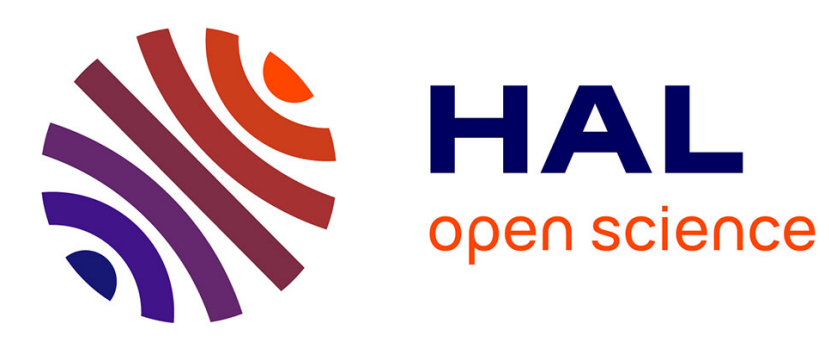

\title{
NORCAMA: Change analysis in SAR time series by likelihood ratio change matrix clustering
}

Xin Su, Charles-Alban Deledalle, Florence Tupin, Hong Sun

\section{To cite this version:}

Xin Su, Charles-Alban Deledalle, Florence Tupin, Hong Sun. NORCAMA: Change analysis in SAR time series by likelihood ratio change matrix clustering. ISPRS Journal of Photogrammetry and Remote Sensing, 2015, 101, pp.247-261. hal-01185740

\section{HAL Id: hal-01185740 https://hal-imt.archives-ouvertes.fr/hal-01185740}

Submitted on 21 Aug 2015

HAL is a multi-disciplinary open access archive for the deposit and dissemination of scientific research documents, whether they are published or not. The documents may come from teaching and research institutions in France or abroad, or from public or private research centers.
L'archive ouverte pluridisciplinaire HAL, est destinée au dépôt et à la diffusion de documents scientifiques de niveau recherche, publiés ou non, émanant des établissements d'enseignement et de recherche français ou étrangers, des laboratoires publics ou privés. 


\title{
NORCAMA: Change Analysis in SAR Time Series by Likelihood Ratio Change Matrix Clustering
}

\author{
Xin $\mathrm{Su}^{1}$, Charles-Alban Deledalle ${ }^{2}$, Florence Tupin ${ }^{1}$ and Hong Sun ${ }^{3}$ \\ 1 LTCI, Institut Mines-Télécom, Télécom ParisTech, Paris, France \\ 2 IMB, CNRS-Université Bordeaux 1, Talence, France \\ ${ }^{3}$ EIS, Wuhan University, Wuhan, P. R. China
}

\begin{abstract}
This paper presents a likelihood ratio test based method of change detection and classification for synthetic aperture radar (SAR) time series, namely NORmalized Cut on chAnge criterion MAtrix (NORCAMA). This method involves three steps: 1) multi-temporal pre-denoising step over the whole image series to reduce the effect of the speckle noise; 2) likelihood ratio test based change criteria between two images using both the original noisy images and the denoised images; 3) change classification by a normalized cut based clustering-and-recognizing method on change criterion matrix (CCM). The experiments on both synthetic and real SAR image series show the effective performance of the proposed framework.
\end{abstract}

Keywords: Change detection, Change classification, SAR time series, Change criterion matrix, Normalized cut, Likelihood ratio test

\section{Notations}

\section{Images:}

$y_{t} \quad$ Noisy image acquired at time $t$;

$\left\{y_{t_{1}}, y_{t_{2}}, \ldots, y_{t_{N}}\right\} \quad$ Noisy multi-temporal images;

$u_{t} \quad$ Noise-free image of $y_{t}$;

$\hat{u}_{t} \quad$ Denoised image of $y_{t}$;

$\hat{u}_{t}^{\mathrm{PPB}} \quad$ Denoised image of $y_{t}$ by PPB filter;

$y_{t}^{1 \text { st }} \quad$ Output of the first (temporal) step in the proposed filter; 


\section{Pixels:}

$y_{t}(i)$

$y_{t}(i+k)$

$u_{t}(i)$

$\hat{u}_{t}(i)$

$\hat{u}_{t}(i+k)$

$y_{m}, y_{n}$

Patches:

$\mathbf{y}_{t}(i)$

$\mathbf{u}_{t}(i)$

$\hat{\mathbf{u}}_{t}(i)$

Pixel at position $i$ in image $y_{t}$;

Pixel at position $i+k$, the $k$-th neighbor of pixel $y_{t}(i)$ in patch $\mathbf{y}_{t}(i)$ (in image $y_{t}$ ) with $k=$ $\{1,2, \ldots, K\}$;

Noise-free pixel at position $i$ in image $u_{t}$;

Denoised pixel at position $i$ in image $\hat{u}_{t}$;

Denoised pixel at position $i+k$, the $k$-th neighbor of pixel $\hat{u}_{t}(i)$ in patch $\hat{\mathbf{u}}_{t}(i+k)$ (in image $\left.\hat{u}_{t}\right)$ with $k=\{1,2, \ldots, K\}$;

Abbreviations of $y_{t_{m}}(i)$ and $y_{t_{n}}(i)$;

Patch of size $\sqrt{K} \times \sqrt{K}$ with pixel $y_{t}(i)$ as center in image $y_{t}$;

Noise-free patch of size $\sqrt{K} \times \sqrt{K}$ with pixel $u_{t}(i)$ as center in image $u_{t}$;

Denoised Patch of size $\sqrt{K} \times \sqrt{K}$ with pixel $\hat{u}_{t}(i)$ as center in image $\hat{u}_{t}$;

\section{Number of looks:}

$L_{t}$

$L_{t}(i)$

$\mathbf{L}_{t}(i)$

$\hat{\mathbf{L}}_{t}(i)$

$\hat{L}_{t}$

Examples:

$y(1)$

$y(2)$

$u(1)$

$\hat{u}(1)$

$L(1)$

$\hat{L}(1)$
Map of number of looks of image $y_{t}$;

Number of looks of pixel $y_{t}(i)$;

Patch of number of looks of patch $\mathbf{y}_{t}(i)$;

Patch of (equivalent) number of looks of patch $\hat{\mathbf{u}}_{t}(i)$

Map of (equivalent) number of looks of image $\hat{u}_{t}$;

An example of pixel (it can be any pixel in the multi-temporal images);

Another example of pixel;

Noise-free pixel of pixel $y(1)$;

Denoised pixel of $y(1)$;

Number of looks of pixel $y(1)$;

Number (equivalent) number of looks of $\hat{u}(1)$. 


\section{Introduction}

Change analysis in remote sensing images is the process of analyzing differences (including identifying, recognizing and so on) in regions of interest by observing them at different dates (Singh, 1989). Many applications of remote sensing images involve change analysis, such as rapid mapping of disaster, land-use and land-cover monitoring and so on. Lu et al. (2011) used a change detection method to detect and locate the landslides for rapid mapping. Similarly, a multi-sensor change detection method between optical and synthetic aperture radar (SAR) imagery is proposed in (Brunner et al., 2010) for earthquake damage assessment of buildings. For urbanization monitoring, post-classification change detection methods are proposed (Taubenböck et al., 2012; Yin et al., 2011). From a methodological point of view, change analysis methods can be classified into two classes, binary-temporal change analysis and multi-temporal change analysis according to the number of images.

In the binary-date change analysis of two optical images, the most widely used operator is difference operator (Singh, 1989). For multi-spectral images, change vector analysis (Bruzzone and Prieto, 2000) is proposed. People also perform the analysis on the transformed data instead of the spectral data directly, such as Tasseled Cap transformation (Fung, 1990; Huang et al., 2002), principal component analysis (Fung and LeDrew, 1987; Deng et al., 2008) and independent component analysis (Marchesi and Bruzzone, 2009). Beyond change detection, Bruzzone and Serpico (1997) explicitly identified land-cover transitions (changes among Bare soil, Corn, Soybean, Sugar beet, Wheat) in multi-temporal remote-sensing images based on supervised classification. Given SAR images, two main approaches have been developed in the literature for change analysis: coherent change detection and incoherent change detection. The former uses the phase information in the SAR images through the study of the coherence map (Preiss and Stacy, 2006). In incoherent change detection, the amplitude log-ratio (Rignot and van Zyl, 1993) is the most common operator. Improvements have been proposed thanks to automatic thresholding methods (Bazi et al., 2005) or multi-scale analysis to preserve details (Bovolo and Bruzzone, 2005). Lombardo and Oliver (2001) proposed a generalized likelihood ratio test given by the ratio between geometric and arithmetic means for SAR images. Quin et al. (2013) extended this ratio of different means to a more general way with an adaptive and nonlinear threshold, which can be applied to not only SAR image pairs but 


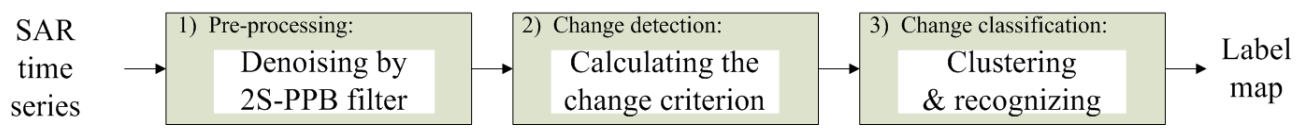

Figure 1: The global diagram of the proposed framework NORmalized Cut on chAnge criterion MAtrix (NORCAMA). It consists of 3 steps, from left to right: 1) the preprocessing step using a multi-temporal SAR filter (2S-PPB) ( $\mathrm{Su}$ et al., 2014) to denoise; 2) a change detection step using the proposed change criteria based on likelihood ratio test; 3) a change classification by the proposed clustering-and-recognizing method.

also SAR time series.

Beyond change analysis between two dates, multi-temporal change analysis (more than 2 dates) mainly focuses on the long-term change information. SAR image features consisting of long-term coherence and temporal backscattering is proposed for a classification purpose (Bruzzone et al., 2004). Julea et al. (2011, 2012) propose a crop monitoring using satellite image time series by a frequent sequential pattern (a group of pixels sharing common temporal patterns and satisfying a minimum spatial connectivity). A generic change detection approach is proposed in (Verbesselt et al., 2010a) for multi-temporal images by detecting and characterizing breaks for additive seasonal and trend changes. It integrates the decomposition of time series into trend, seasonal, and remaining components within a long-term time series. An improved harmonic seasonal model which requires fewer observations has been presented in (Verbesselt et al., 2010b). Transform tools (De Jong et al., 2011; Martínez and Gilabert, 2009) have also been used for analysis of the normalized difference vegetation index time series.

In this paper, we address the problem of change classification of multitemporal SAR series. We will focus on same-sensor same-incidence case and consider binary changes between two dates with applications to urban areas. Although this is a restrictive case, this approach could be seen as a screening stage for improved change analysis taking into account continuous evolution. A global processing chain in 3 steps, namely NORmalized Cut on chAnge criterion MAtrix (NORCAMA), is defined, as shown in Fig.1. In the pre-processing step, a multi-temporal SAR image filter is used to reduce speckle phenomenon. After that, two change criteria based on likelihood ratio test combining noisy and denoised data are developed and compared. The final step is a clustering-and-recognizing classification based on the change criterion matrix, in which changes are classified into different types (including step change, impulse change, cycle change and complex change). The last 2 
steps as well as the global framework are the main contribution of this paper. Evaluation on synthetic and real images show the good performance of the proposed approach.

This paper is organized as follows. Section 2 briefly recalls the multitemporal denoising method for SAR images. The proposed approximate likelihood ratio test and generalized likelihood ratio test change criteria are presented in Section 3. The proposed clustering-and-recognizing change classification method is then detailed in Section 4. This is followed by evaluation (Section 5) and conclusion (Section 6).

\section{Pre-Processing: Multi-Temporal Denoising}

When dealing with multi-temporal images, lots of information is available and useful for estimation purposes in the time series. However, the presence of high fluctuations due to speckle in SAR images hampers their analysis. Based on this motive, we proposed a two-step probabilistic patch based (2SPPB) denoising method (Su et al., 2014) relying on non local means (Buades et al., 2005) and probabilistic patch based weights (PPB) (Deledalle et al., 2009) adapted to multi-temporal SAR images. To allow a self-content reading of this paper and to introduction the useful notations, we briefly summarize the main steps of this approach. A complete description can be found in ( $\mathrm{Su}$ et al., 2014). It consists of a temporal averaging step and a spatial denoising step (summarized in Algorithm 1). Firstly, an average image is created by combining stable pixels while keeping unchanged the pixels not in accordance with the other dates (temporal averaging step). Then, on this improved image, a spatial denoising step is applied. A key point in both the temporal and spatial averaging is the weights based on the similarity between pixels, which are measured by similarity between surrounding patches. This section presents a brief summary of 2SPPB which will be useful for the following steps.

\subsection{Pixel similarity}

We denote by $y_{t}$ the observed SAR image, by $y_{t}(i)$ the noisy intensity value at pixel index $i$ at time $t$, and by $\hat{u}_{t}(i)$ the estimation of the actual pixel value $u_{t}(i)$ (the true value that we are looking for). Considering $\left\{y_{t_{1}}, y_{t_{2}}, \ldots, y_{t_{N}}\right\}$ as the stack of multi-temporal images, the similarity criterion $S\left(y_{t}(i), y_{t^{\prime}}(i), h, h^{\prime}\right)$ between pixels $y_{t}(i)$ and $y_{t^{\prime}}(i)$ is defined through 
the similarity of their surrounding patches $\mathbf{y}_{t}(i)$ and $\mathbf{y}_{t^{\prime}}(i)$ (the lower, the more similar they are supposed to be):

$$
S\left(y_{t}(i), y_{t^{\prime}}(i), h, h^{\prime}\right)=\frac{S_{\mathrm{GLR}}\left[\mathbf{y}_{t}(i), \mathbf{y}_{t^{\prime}}(i)\right]}{h}+\frac{S_{\mathrm{KL}}\left[\hat{\mathbf{u}}_{t}(i), \hat{\mathbf{u}}_{t^{\prime}}(i)\right]}{h^{\prime}}
$$

$S\left[y_{t}(i), y_{t^{\prime}}(i), h, h^{\prime}\right]$ consists of the sum of a generalized likelihood ratio $S_{\mathrm{GLR}}$ (GLR) from noisy images $y_{t}$ and $y_{t^{\prime}}$ and a Kullback-Leibler divergence $S_{\mathrm{KL}}$ (KL) from currently denoised images $\hat{u}_{t}$ and $\hat{u}_{t^{\prime}}$. $S_{\mathrm{GLR}}$ and $S_{\mathrm{KL}}$ are normalized by parameters $h$ and $h^{\prime}$. For any pair of pixels $y(1)$ and $y(2)$, the GLR criterion $S_{\mathrm{GLR}}$ is comparing two small square patches $\mathbf{y}(1)$ and $\mathbf{y}(2)$ of size $K$ surrounding pixels at the positions of $y(1)$ and $y(2)$, and is defined as ( $\mathrm{Su}$ et al., 2014):

$$
\begin{aligned}
& S_{\mathrm{GLR}}[\mathbf{y}(1), \mathbf{y}(2)]= \\
& \quad \sum_{k \in K}[L(1+k)+L(2+k)] \log [L(1+k) y(1+k)+L(2+k) y(2+k)] \\
& \quad-[L(1+k)+L(2+k)) \log (L(1+k)+L(2+k)] \\
& \quad-L(1+k) \log (y(1+k))-L(2+k) \log (y(2+k))
\end{aligned}
$$

where, $y(1+k)$ is $k$-pixel in patch $\mathbf{y}(1)$ and $L(1+k)$ is the (equivalent) number of looks of $y(1+k)$ (idem for $y(2+k)$ and $L(2+k)$ ). The KL criterion $S_{\mathrm{KL}}$ is computed from a pair of denoised results $\hat{u}_{t}$ and $\hat{u}_{t^{\prime}}$ of the noisy image $y_{t}$ and $y_{t^{\prime}}$ respectively. The criterion $S_{\mathrm{KL}}$ is also defined on two patches $\hat{\mathbf{u}}(1)$ and $\hat{\mathbf{u}}(2)$ as (Su et al., 2014):

$$
\begin{aligned}
& S_{\mathrm{KL}}[\hat{\mathbf{u}}(1), \hat{\mathbf{u}}(2)]= \\
& \quad \sum_{k \in K} L(1+k) \frac{\hat{u}(2+k)}{\hat{u}(1+k)}+L(2+k) \frac{\hat{u}(1+k)}{\hat{u}(2+k)}-L(1+k)-L(2+k) \\
& \quad+L(1+k)[\psi(L(1+k))-\psi(L(2+k))+\ln (\hat{u}(1+k))+\ln (\hat{u}(2+k))] \\
& \quad-L(2+k)[\psi(L(1+k))-\psi(L(2+k))+\ln (\hat{u}(1+k))+\ln (\hat{u}(2+k))]
\end{aligned}
$$

where $\psi(\dot{)}$ is the digamma function. 


\subsection{Two steps denoising}

The first step of $2 \mathrm{SPPB}$ is to average the temporal pixels with binary weights:

$$
\begin{gathered}
y_{t}^{1 \mathrm{st}}(i)=\frac{1}{Z} \sum_{t^{\prime} \in\left[t_{1}, t_{N}\right]} \varphi\left[S\left(y_{t}(i), y_{t^{\prime}}(i), h_{1 \mathrm{st}}, h_{1 \mathrm{st}}^{\prime}\right)\right] \cdot y_{t^{\prime}}(i) \\
\text { with, } Z=\sum_{t^{\prime} \in\left[t_{1}, t_{N}\right]} \varphi\left[S\left(y_{t}(i), y_{t^{\prime}}(i), h_{1 \mathrm{st}}, h_{1 \mathrm{st}}^{\prime}\right)\right] \\
\varphi\left[S\left(y_{t}(i), y_{t^{\prime}}(i), h_{1 \mathrm{st}}, h_{1 \mathrm{st}}^{\prime}\right)\right]= \begin{cases}1, & \text { if } S\left(y_{t}(i), y_{t^{\prime}}(i), h_{1 \mathrm{st}}, h_{1 \mathrm{st}}^{\prime}\right)<1 \\
0, & \text { otherwise }\end{cases}
\end{gathered}
$$

where, $S\left(y_{t}(i), y_{t^{\prime}}(i), h_{1 \mathrm{st}}, h_{1 \mathrm{st}}^{\prime}\right)$ is computed from noisy images $y_{t}(i)$ and $y_{t^{\prime}}(i)$, and denoised images by PPB. The quantity $Z$ is the weight normalizing parameter. The second step of 2SPPB approach is to exploit similar pixels in the temporally average image $y_{t_{t}}^{1 \text { st }}$ rather than in the stack $\left\{y_{t_{1}}, \ldots, y_{t_{N}}\right\}$. The estimation at time $t$ is thus given by

$$
\begin{gathered}
\hat{u}_{t}(i)=\frac{1}{Z} \sum_{j \in \Omega_{i}} \exp \left[-S\left(y_{t}(i), y_{t^{\prime}}(i), h_{2 \mathrm{nd}}, h_{2 \mathrm{nd}}^{\prime}\right)\right] \cdot y_{t}^{1 \mathrm{st}}(j) \\
Z=\sum_{j \in \Omega_{i}} \exp \left[-S\left(y_{t}(i), y_{t^{\prime}}(i), h_{2 \mathrm{nd}}, h_{2 \mathrm{nd}}^{\prime}\right)\right]
\end{gathered}
$$

where the GLR criterion in $S\left(y_{t}(i), y_{t^{\prime}}(i), h_{2 \text { nd }}, h_{2 \text { nd }}^{\prime}\right)$ is computed using the temporal step (the first step) result $y_{t_{t}}^{\text {1st }}$. To improve the final $\hat{u}_{t}$ estimation, the KL criterion is iteratively refined by using the previous estimate. According to (Deledalle et al., 2009) and (Su et al., 2014), the number of iterations is set to 10. The final result of this temporal denoising step will be denoted by $\hat{u}_{t}$ for date $t$ (with a map of corresponding number of looks $\hat{L}_{t}$ ). Algorithm 1 summarizes the multi-temporal denoising processing. 


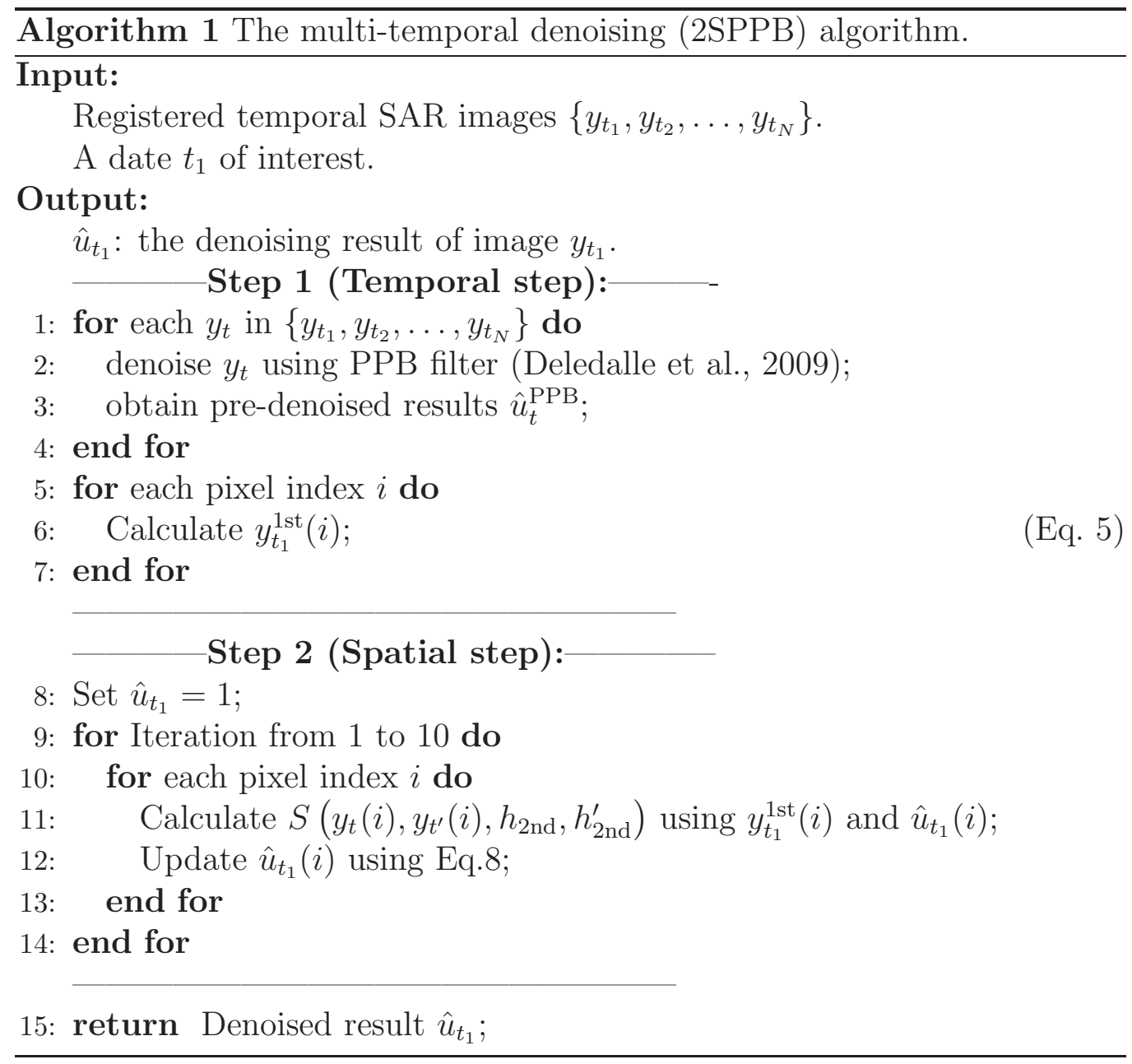

\section{Change Detection}

In statistics, change detection problem can be considered as a comparison of two hypotheses $\mathcal{H}_{0}$ and $\mathcal{H}_{1}$ (Radke et al., 2005):

$$
\begin{aligned}
& \mathcal{H}_{0}: u(1)=u(2)=u(12) \text { (null hypothesis) } \\
& \mathcal{H}_{1}: u(1) \neq u(2) \text { (alternative hypothesis) }
\end{aligned}
$$

where $\mathcal{H}_{0}$ is unchanged case and hypothesis $\mathcal{H}_{1}$ is changed case. Likelihood ratio tests are classical techniques that can be used here to decide between both of our hypotheses by thresholding the response of the following likeli- 
hood ratio (see e.g., (Kay, 1998)):

$$
\mathcal{R}(\mathcal{Y})=\frac{p\left(\mathcal{Y} \mid u(12), \mathcal{H}_{0}\right)}{p\left(\mathcal{Y} \mid u(1), u(2), \mathcal{H}_{1}\right)}
$$

where $\mathcal{Y}$ is an observation or a set of observations that typically depends on $u(1)$ and $u(2)$. In this section, we propose to develop the likelihood ratio test using the multi-temporal denoising results. Contrary to most likelihood ratio tests which only use noisy data, both the denoised data and the noisy data are involved in the proposed criteria.

\subsection{Change Criterion by Approximate Likelihood Ratio Test}

Recall that by $y$ we denote a pixel intensity value. Under the speckle noise model described in (Goodman, 1976), $y$ is a realization of a random number characterized by the Gamma probability density function (pdf) $p(y \mid u)$. The quantity $u$ is the parameter of this Gamma pdf denoted as the noise-free pixel value. According to Eq.10, the change criterion between $y(1)$ and $y(2)$ using likelihood ratio can be defined as:

$$
\mathcal{R}^{\mathrm{ALRT}}(y(1), y(2))=\frac{p\left(y(1), y(2) \mid u(12), \mathcal{H}_{0}\right)}{p\left(y(1), y(2) \mid u(1), u(2), \mathcal{H}_{1}\right)}
$$

The criterion in Eq. 11 is a composite hypothesis problem because it requires the noise-free value $u$ which is unavailable in practice. Instead of using usual generalized likelihood ratio extensions, we propose to use an approximation which replaces the unknown noise free $u$ by their estimated values $\hat{u}$. Combined with the Gamma probability density function, the likelihood ratio in Eq. 10 becomes:

$$
\begin{aligned}
\mathcal{R}^{\mathrm{ALRT}}(y(1), y(2))= & \frac{p\left(y(1), y(2) \mid u(12), \mathcal{H}_{0}\right)}{p\left(y(1), y(2) \mid u(1), u(2), \mathcal{H}_{1}\right)} \\
= & {\left[\frac{1}{4}\left(\frac{\hat{u}(2)}{\hat{u}(1)}+\frac{\hat{u}(1)}{\hat{u}(2)}+2\right)\right]^{-L} } \\
& \exp \left[L\left(\frac{y(1)}{\hat{u}(1)}+\frac{y(2)}{\hat{u}(2)}-\frac{2 y(1)+2 y(2)}{\hat{u}(1)+\hat{u}(1)}\right)\right]
\end{aligned}
$$

where $L=L(1)=L(2)$ is the original spatially-invariant (equivalent) number of looks. Note that the approximate likelihood ratio $\mathcal{R}^{\text {ALRT }}$ highly depends 
on the denoised values $\hat{u}(1)$ and $\hat{u}(2)$, since $\mathcal{R}^{\mathrm{ALRT}}(y(1), y(2)) \equiv 1$ when $\hat{u}(1)=\hat{u}(2)$ whatever $y(1)$ and $y(2)$.

\subsection{Change Criterion by Generalized Likelihood Ratio Test}

In a more general way, we can take into account the denoised values and consider the likelihood probability of $\mathcal{H}_{0}$ and $\mathcal{H}_{1}$ as $p\left(y(1), y(2), \hat{u}(1), \hat{u}(2) \mid \mathcal{H}_{0}\right)$ and $p\left(y(1), y(2), \hat{u}(1), \hat{u}(2) \mid \mathcal{H}_{1}\right)$. To simplify this likelihood probability, we can assume that $\{y(1), \hat{u}(1)\}$ and $\{y(2), \hat{u}(2)\}$ are independent, although this assumption is not well justified (since, typically $y(1)$ can intervene in the estimation of $\hat{u}(2))$. Thus,

$$
\begin{aligned}
& p\left(y(1), y(2), \hat{u}(1), \hat{u}(2) \mid \mathcal{H}_{0}\right)=p\left(y(1), \hat{u}(1) \mid u(12), \mathcal{H}_{0}\right) p\left(y(2), \hat{u}(2) \mid u(12), \mathcal{H}_{0}\right) \\
& p\left(y(1), y(2), \hat{u}(1), \hat{u}(2) \mid \mathcal{H}_{1}\right)=p\left(y(1), \hat{u}(1) \mid u_{1}, \mathcal{H}_{1}\right) p\left(y(2), \hat{u}(2) \mid u_{2}, \mathcal{H}_{1}\right) .
\end{aligned}
$$

The likelihood ratio test is given by:

$$
\mathcal{R}^{\mathrm{GLRT}}(y(1), y(2))=\frac{p\left(y(1), \hat{u}(1) \mid u(12), \mathcal{H}_{0}\right) p\left(y(2), \hat{u}(2) \mid u(12), \mathcal{H}_{0}\right)}{p\left(y(1), \hat{u}(1) \mid u(1), \mathcal{H}_{1}\right) p\left(y(2), \hat{u}(2) \mid u(2), \mathcal{H}_{1}\right)} .
$$

Since $u(12), u_{1}$ and $u_{2}$ are not available, they can be replaced by their maximum likelihood (ML) estimation:

$$
\begin{aligned}
& u_{1}=\frac{L y(1)+\hat{L}(1) \hat{u}(1)}{L+\hat{L}(1)} \\
& u_{2}=\frac{L y(2)+\hat{L}(2) \hat{u}(2)}{L+\hat{L}(2)} \\
& u(12)=\frac{L y(1)+L y(2)+\hat{L}(1) \hat{u}(1)+\hat{L}(2) \hat{u}(2)}{2 L+\hat{L}(1)+\hat{L}(2)} .
\end{aligned}
$$

$L=y(1)=y(2), \hat{L}(1)$ and $\hat{L}(2)$ are the number of looks of $y(1), \hat{u}(1)$ and $\hat{u}(2)$ respectively. Note that this is very similar to (Lombardo and Oliver, 2001). Nevertheless, the multi-temporal denoised values used in the proposed approach can provide more accurate estimation without loss of spatial resolution. It was not the case in (Lombardo and Oliver, 2001) where spatial partitioning and averaging were introduced as post-processing steps. In case of Gamma distributions with different number of looks, each probability term $p(y, \hat{u} \mid u)$ in Eq.13 can be approximated under conditional independence 
assumption by:

$$
\begin{aligned}
& p(y, \hat{u} \mid u)=p(y \mid u) p(\hat{u} \mid u) \\
& \quad=\frac{y^{-1} \hat{u}^{-1}}{\Gamma(L) \Gamma(\hat{L})} \frac{(L y)^{L}(\hat{L} \hat{u})^{\hat{L}}}{u^{L+\hat{L}}} \exp \left(-\frac{L y+\hat{L} \hat{u}}{u}\right) .
\end{aligned}
$$

Finally, the change criterion given by the generalized likelihood boils down to:

$$
\begin{aligned}
\mathcal{R}^{\mathrm{GLRT}}(y(1), y(2))= & \left(\frac{L y(1)+\hat{L}(1) \hat{u}(1)}{L+\hat{L}(1)}\right)^{L+\hat{L}(1)}\left(\frac{L y(2)+\hat{L}(2) \hat{u}(2)}{L+\hat{L}(2)}\right)^{L+\hat{L}(2)} \\
& \left(\frac{2 L+\hat{L}(1)+\hat{L}(2)}{L y(1)+\hat{L}(1) \hat{u}(1)+L y(2)+\hat{L}(2) \hat{u}(2)}\right)^{2 L+\hat{L}(1)+\hat{L}(2)}
\end{aligned}
$$

Unlike $\mathcal{R}^{\mathrm{ALRT}}$, the generalized likelihood ratio $\mathcal{R}^{\mathrm{GLRT}}$ does not rely much on the denoised values $\hat{u}(1)$ and $\hat{u}(2)$. Indeed, even though $\hat{u}(1)=\hat{u}(2), \mathcal{R}^{\text {GLRT }}$ still depends on the noisy values $y(1)$ and $y(2)$.

\subsection{Thresholds for Change Detection}

In (Kervrann and Boulanger, 2006), the authors proposed to define the parameters according to the quantiles of the similarity criterion when it is subject to identical and independent distributed random variables. Pursuing this idea, we propose to choose the thresholds according to the quantiles of $\mathcal{R}^{\mathrm{ALRT}}$ and $\mathcal{R}^{\mathrm{GLRT}}$. The change detection threshold can be set by $\tau^{\mathrm{ALRT}}=$ quantile $\left(\mathcal{R}^{\mathrm{ALRT}}, \alpha=0.01\right.$ ) (and $\tau^{\mathrm{GLRT}}=$ quantile $\left(\mathcal{R}^{\mathrm{GLRT}}, \alpha=0.01\right)$ ), which means the false alarm rate is $1 \%$. However, it is not easy to obtain the distribution of $\mathcal{R}^{\mathrm{ALRT}}$ and $\mathcal{R}^{\mathrm{GLRT}}$ since they depend on the number of looks of noisy images, the number of images used in the denoising process and all the parameters of multi-temporal filter (such as the $h, h^{\prime}$, search window size, patch size and so on). Thus, $\mathcal{R}^{\mathrm{ALRT}}$ and $\mathcal{R}^{\mathrm{GLRT}}$ distributions are simulated using synthetic SAR images to choose the thresholds.

For our purpose of parameter setting, any picture can be used to generate multiple speckle images with no change. Note that all the synthetic multitemporal noisy images use the same true image, which guarantees no changes among them. The same number of images and the same number of looks as 


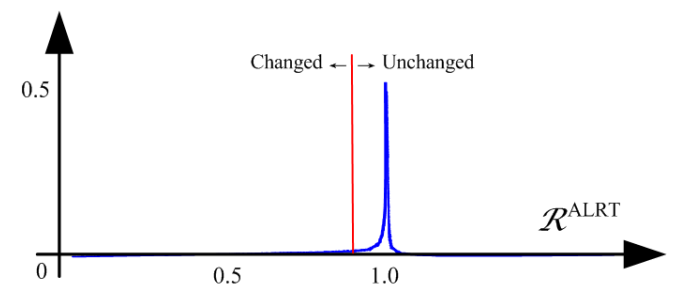

(a) $\mathcal{R}^{\text {ALRT }}$

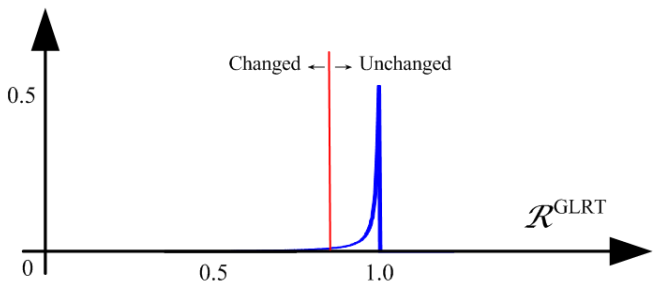

(b) $\mathcal{R}^{\mathrm{GLRT}}$

Figure 2: The simulated histograms of $\mathcal{R}^{\mathrm{ALRT}}$ and $\mathcal{R}^{\text {GLRT }}$ using synthetic images. The red lines are thresholds $\tau^{\text {ALRT }}$ and $\tau^{\text {GLRT }}$ with false alarm $1 \%$. The blue lines are the histograms of unchanged $\mathcal{R}^{\mathrm{ALRT}}$ and $\mathcal{R}^{\mathrm{GLRT}}$.

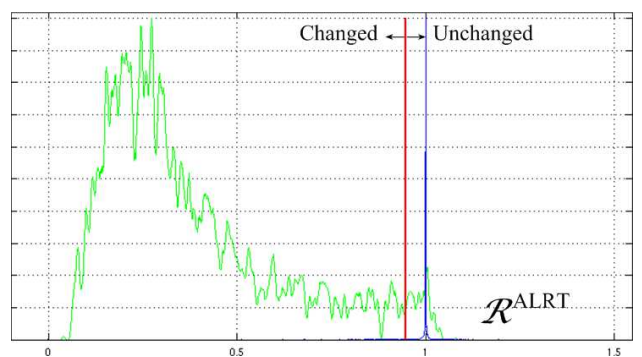

(a) $\mathcal{R}^{\text {ALRT }}$

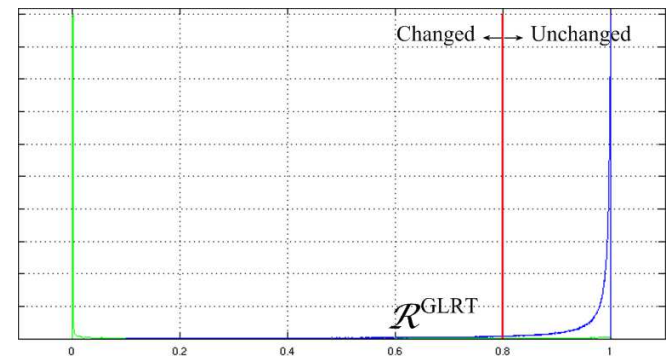

(b) $\mathcal{R}^{\mathrm{GLRT}}$

Figure 3: An example of the normalized histograms (peak normalization) of $\mathcal{R}^{\mathrm{ALRT}}$ and $\mathcal{R}^{\text {GLRT }}$ using real SAR images Paris (detailed in section 5). The red lines are thresholds $\tau^{\mathrm{ALRT}}$ and $\tau^{\mathrm{GLRT}}$ with false alarm $1 \%$. The blue lines are the histograms of unchanged $\mathcal{R}^{\mathrm{ALRT}}$ and $\mathcal{R}^{\mathrm{GLRT}}$. The green lines are the histograms of changed $\mathcal{R}^{\mathrm{ALRT}}$ and $\mathcal{R}^{\mathrm{GLRT}}$.

the real SAR images to be processed have to be used. Then, the multitemporal denoising process in section 2 is performed. The approximate and generalized likelihood ratio test change criteria calculated from these images are considered as pure distributions of $\mathcal{R}^{\mathrm{ALRT}}$ and $\mathcal{R}^{\mathrm{GLRT}}$. As shown in Fig.2, the histograms of $\mathcal{R}^{\mathrm{ALRT}}$ and $\mathcal{R}^{\mathrm{GLRT}}$ are truncated by the thresholds (red lines) with false alarm 1\%. The parts on the right of the thresholds are considered as unchanged case, the left part is changed case. Fig.3 shows the $\mathcal{R}^{\mathrm{ALRT}}$ and $\mathcal{R}^{\mathrm{GLRT}}$ histograms of changed and unchanged pixels in real SAR data Paris (image information detailed in section 5). Those changed pixels are labeled manually. The robustness of the proposed change criteria $\mathcal{R}^{\mathrm{ALRT}}$ and $\mathcal{R}^{\mathrm{GLRT}}$ can be epitomized by the overlap of $\mathcal{R}^{\mathrm{ALRT}}$ and $\mathcal{R}^{\mathrm{GLRT}}$ histograms. 

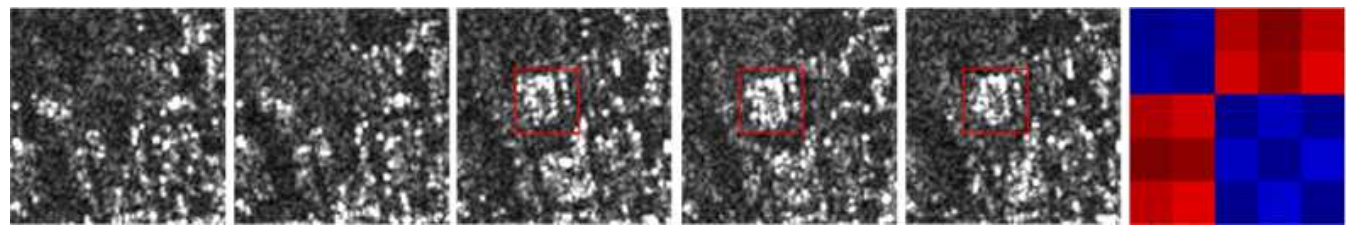

(a) Step change.
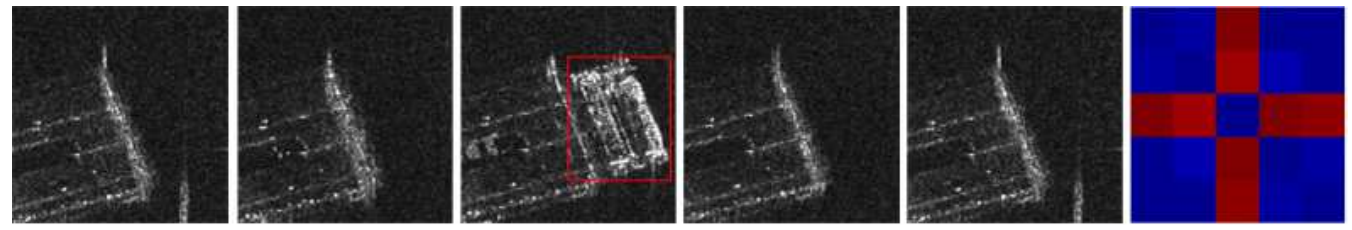

(b) Impulse change.

Figure 4: Examples of step changes and impulse changes. From left to right: original multi-temporal SAR images at time $t_{1}, t_{2}, t_{3}, t_{4}, t_{5}$, change criterion matrix of a pixel in the red rectangle (cold color: unchanged; warm color: changed).

\section{Change Classification}

Change detection between 2 dates aims at detecting a binary pattern (change or no-change). When dealing with a multi-temporal data set (more than 2 dates), the analysis among them is much more complex. As said in the introduction, we will focus in this work on binary changes which means that we will not take into account continuous changes. This is well adapted for few time series and urban applications. This approach can also be seen as a preliminary step of screening before improved classification of continuous changes. For instance, the temporal behaviors of a new building usually can be considered as a step change, which means that comparing the oldest date with other dates, it was unchanged at the beginning but it changed since a certain date (shown in Fig. 4.a). Similarly, we can define the boats in rivers or cars on the roads as impulse changes (Fig. 4.b). These change information can be used in the multi-temporal image interpretation tasks. Therefore, a clustering-and-recognizing method is proposed to classify changes into different types. This method consists of two steps, clustering using normalized cut on a change criterion matrix (to assign a same label to similar or unchanged temporal pixels) and classification according to their temporal behaviors. The following subsections detail the proposed approach. 


\subsection{Change Criterion Matrix (CCM)}

At position $i$ of a multi-temporal SAR series $\left\{y_{t_{1}}, \ldots, y_{t_{N}}\right\}$, we have the two pixel series $\left\{y_{t_{1}}(i), \ldots, y_{t_{N}}(i)\right\}$ (original noisy data), $\left\{\hat{u}_{t_{1}}(i), \ldots, \hat{u}_{t_{N}}(i)\right\}$ (denoised data by multi-temporal filter of section 2 ) and associated equivalent number of looks $\left\{L_{t_{1}}(i), \ldots, L_{t_{N}}(i)\right\}$. The change criterion matrix (CCM) at position $i$ is defined as:

$$
\mathcal{M}(i)=\left[\begin{array}{cccc}
\mathcal{R}\left(y_{1}, y_{1}\right) & \mathcal{R}\left(y_{1}, y_{2}\right) & \ldots & \mathcal{R}\left(y_{1}, y_{N}\right) \\
\mathcal{R}\left(y_{2}, y_{1}\right) & \mathcal{R}\left(y_{2}, y_{2}\right) & \ldots & \mathcal{R}\left(y_{2}, y_{N}\right) \\
\ldots & \ldots & \ldots & \ldots \\
\mathcal{R}\left(y_{N}, y_{1}\right) & \mathcal{R}\left(y_{N}, y_{2}\right) & \ldots & \mathcal{R}\left(y_{N}, y_{N}\right)
\end{array}\right]
$$

where $\mathcal{R}\left(y_{n}, y_{m}\right)$ shorts for $\mathcal{R}\left[y_{t_{n}}(i), y_{t_{m}}(i)\right]$ and denotes the change criterion $\left(\mathcal{R}^{\mathrm{ALRT}}\right.$ or $\left.\mathcal{R}^{\mathrm{GLRT}}\right)$ between pixel $y_{t_{n}}(i)$ and $y_{t_{m}}(i)$. Note that $\mathcal{R}\left(y_{n}, y_{m}\right)=1$ when $n=m$. Contrary to the multi-date divergence matrix in (Atto et al., 2013) performing at the image or sub-image level, the CCM presents the change information at pixel level. Each $\mathrm{CCM} \mathcal{M}(i)$ denotes the temporal behavior of the pixel series at position $i$.

\subsection{Clustering by Normalized Cut}

Spectral clustering techniques make use of the similarity matrix of the data to perform clustering. Since the CCM can be considered as a similarity matrix of the time series, spectral clustering method has been applied on CCM to cluster the temporal pixels. In this case, no more similarity measurements is needed compared with other clustering methods (like K-Means algorithm for which new similarity to cluster center has to be computed).

Normalized spectral clustering proposed by Shi and Malik (2000) is employed in this work, which can be summarized in Algorithm 2. In this algorithm, the normalized Laplacian matrix $\mathcal{M}^{\mathrm{u}}(i)$ is computed by:

$$
\begin{aligned}
& \mathcal{M}^{\mathrm{u}}(i)=\mathcal{M}^{\mathrm{s}}(i)-\mathcal{M}(i) \\
& \mathcal{M}^{\mathrm{s}}(i)=\left[\begin{array}{cccc}
\sum \mathcal{R}\left(y_{1}, y_{n}\right) & 0 & \ldots & 0 \\
0 & \sum \mathcal{R}\left(y_{2}, y_{n}\right) & \ldots & 0 \\
\ldots & \ldots & \ldots & \ldots \\
0 & 0 & \ldots & \sum \mathcal{R}\left(y_{N}, y_{n}\right)
\end{array}\right] \\
& \sum \mathcal{R}\left(y_{m}, y_{n}\right)=\sum_{n=1, \ldots, N} \mathcal{R}\left(y_{m}, y_{n}\right)
\end{aligned}
$$


The only parameter in Algorithm 2 is the number of clusters $p$. Choosing the number of clusters $p$ is a general problem for all clustering algorithms, and a variety of successful methods have been devised (more details in (Von Luxburg, 2007)). Eigengap heuristic is one of them and particularly designed for spectral clustering. The main idea is to choose the number $p$ such that all eigenvalues $\lambda_{1}, \ldots, \lambda_{p}$ are very small, but $\lambda_{p+1}$ is relatively larger (all eigenvalues are sorted in ascending order). However, this heuristic fails when the clusters of the data are overlapping (because of noise). To solve this problem, we binarize the CCM $\mathcal{M}(i)$ using the change detection threshold.

$$
\begin{gathered}
\mathcal{M}^{\mathrm{b}}(i)=\left[\begin{array}{ccc}
\mathcal{R}^{\mathrm{b}}\left(y_{1}, y_{1}\right) & \ldots & \mathcal{R}^{\mathrm{b}}\left(y_{1}, y_{N}\right) \\
\mathcal{R}^{\mathrm{b}}\left(y_{2}, y_{1}\right) & \ldots & \mathcal{R}^{\mathrm{b}}\left(y_{2}, y_{N}\right) \\
\ldots & \ldots & \ldots \\
\mathcal{R}^{\mathrm{b}}\left(y_{N}, y_{1}\right) & \ldots & \mathcal{R}^{\mathrm{b}}\left(y_{N}, y_{N}\right)
\end{array}\right] \\
\mathcal{R}^{\mathrm{b}}\left(y_{m}, y_{n}\right)= \begin{cases}0 \text { if } \mathcal{R}\left(y_{m}, y_{n}\right)<\tau \\
1 \text { if } \mathcal{R}\left(y_{m}, y_{n}\right) \geqslant \tau\end{cases}
\end{gathered}
$$

The Eigengap heuristic performed on the binary change criterion matrix $\mathcal{M}^{\mathrm{b}}(i)$ can easily be used to estimate the number of clusters $p$ (see the example shown in Fig. 5). The toy model in Fig. 5.a has 2 clusters. Using CCM $\mathcal{M}(i)$ in 5.b, the difference between eigenvalues $\lambda_{2}$ and $\lambda_{3}$ is not large enough compared with the one between $\lambda_{1}$ and $\lambda_{2}$. It is very easy to find the best estimation of $p$ using the binary $\mathrm{CCM} \mathcal{M}^{\mathrm{b}}(i)$ in 5.c because of the large gap between $\lambda_{2}$ and $\lambda_{3}$. It is obvious that this estimation of $p$ highly depends on the choice of the thresholds. However, the robustness of the proposed change criteria (especially $\mathcal{R}^{\mathrm{GLRT}}$ shown in Fig.3) can guaranty the estimation accuracy of $p$.

\subsection{Recognizing}

After clustering, each pixel series $\left\{y_{t_{1}}(i), \ldots, y_{t_{N}}(i)\right\}$ has a cluster label series $\left\{l_{t_{1}}(i), \ldots, l_{t_{N}}(i)\right\}$, in which $l_{t_{n}}(i) \in\{1, \ldots, p\}$. We can identify different types of change according to the transformation in the cluster label series $\left\{l_{t_{1}}(i), \ldots, l_{t_{N}}(i)\right\}$. For example, if $p=1$, there is no change among this pixel series. If $p=2$ with cluster label series $\{1,1, \ldots, 1,2,2, \ldots, 2\}$, it is a step change. Impulse change usually has $p=2$ and cluster label series is $\{1,1, \ldots, 1,2,2, \ldots, 2,1,1, \ldots, 1\}$. When $p \geqslant 3$, the transformation is complex and changes are defined as complex case. According to these identifications (details in Table 2), changes can be classified into several classes. 


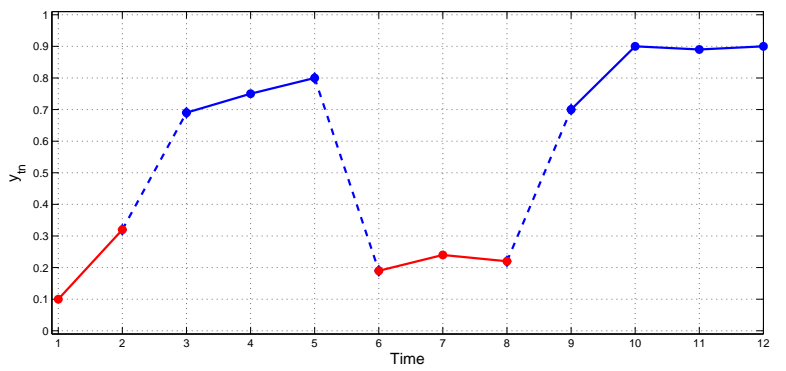

(a) A pixel series $\left\{y_{t_{1}}(i), \ldots, y_{t_{1} 2}(i)\right\}$ which should be clustered into 2 groups (red and blue).
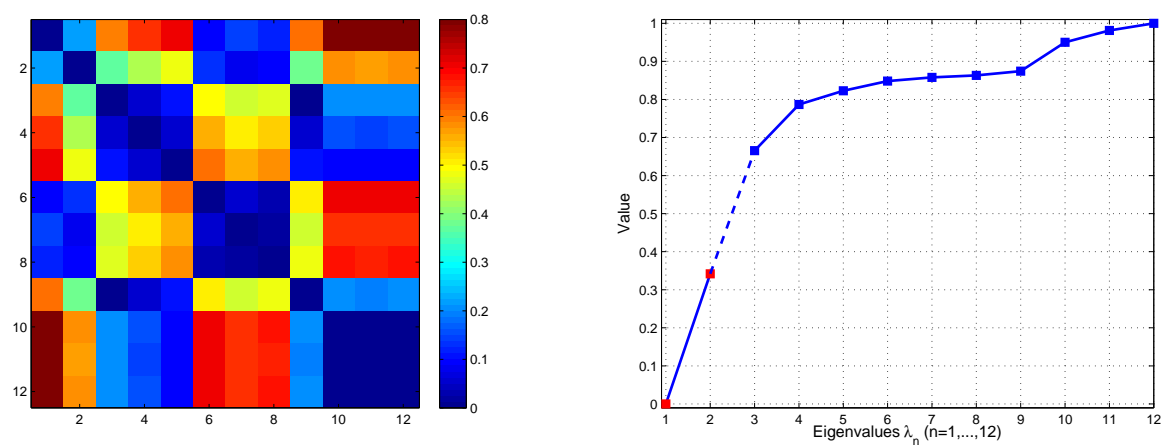

(b) $\operatorname{CCM} \mathcal{M}(i)$ and its eigenvalues.
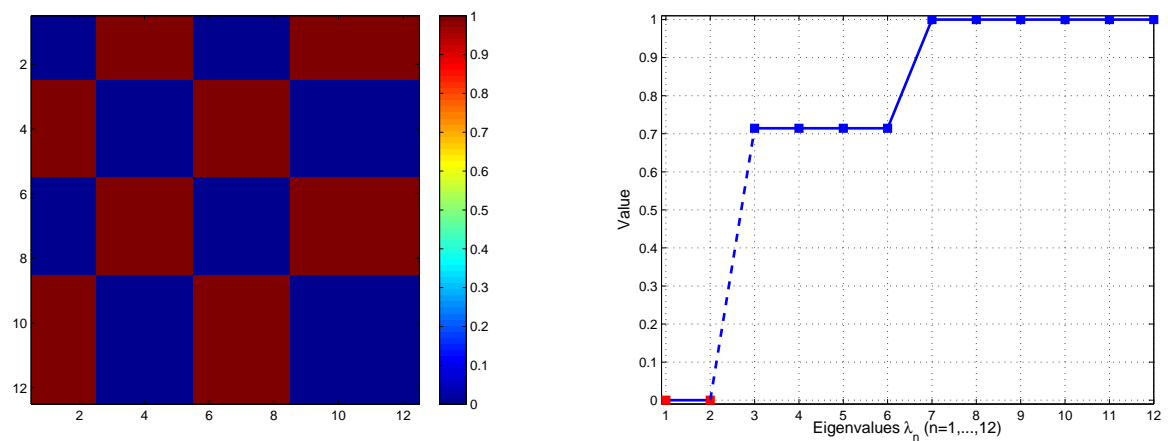

(c) Binary $\mathrm{CCM} \mathcal{M}^{\mathrm{b}}(i)$ and its eigenvalues.

Figure 5: Estimation of number of clusters. (a). a pixel series $\left\{y_{t_{1}}(i), \ldots, y_{t_{1} 2}(i)\right\}$ which should be clustered into 2 groups; (b). Estimation of the number of clusters using CCM $\mathcal{M}(i)$, the gap between $\lambda_{2}$ and $\lambda_{3}$ is not obvious; (b). Estimation of the number of clusters using binary CCM $\mathcal{M}^{\mathrm{b}}(i)$, the gap between $\lambda_{2}$ and $\lambda_{3}$ is larger. 
Algorithm 2 Clustering of the pixel series (Normalized spectral clustering (Shi and Malik, 2000))

\section{Input:}

A change criterion matrix $\mathcal{M}(i)$ of pixel series $\left\{y_{t_{1}}(i), \ldots, y_{t_{N}}(i)\right\}$, number $p$ of clusters to construct.

\section{Output:}

The clustering labels $\left\{l_{t_{1}}(i), \ldots, l_{t_{N}}(i)\right\}$ for pixel series $\left\{y_{t_{1}}(i), \ldots, y_{t_{N}}(i)\right\}$

1: Compute the normalized Laplacian matrix $\mathcal{M}^{\mathrm{u}}(i)$ using Eq.18.

2: Compute the first $p$ generalized eigenvectors $\boldsymbol{v}_{1}, \ldots, \boldsymbol{v}_{p}$ of $\mathcal{M}^{\mathrm{u}}(i)$ $\left(\mathcal{M}^{\mathrm{u}}(i) \boldsymbol{v}=\lambda \boldsymbol{I} \boldsymbol{v}\right)$.

3: Let $\boldsymbol{v}^{\prime}$ be the matrix containing the vectors $\boldsymbol{v}_{1}, \ldots, \boldsymbol{v}_{p}$ as columns.

4: Consider each row of $\boldsymbol{v}^{\prime}$ as a sample, $\boldsymbol{v}^{\prime}=\left\{\boldsymbol{v}_{1}^{\prime}, \ldots, \boldsymbol{v}_{N}^{\prime}\right\}$.

5: Cluster the samples $\boldsymbol{v}_{1}^{\prime}, \ldots, \boldsymbol{v}_{N}^{\prime}$ with the k-means algorithm into clusters with $\mathrm{K}=p$ as the number of clusters. The cluster labels of $\boldsymbol{v}_{1}^{\prime}, \ldots, \boldsymbol{v}_{N}^{\prime}$ are $l_{t_{1}}(i), \ldots, l_{t_{N}}(i)\left(l_{t_{n}}(i) \in\{1, \ldots, p\}\right)$.

6: return Cluster labels $l_{t_{1}}(i), \ldots, l_{t_{N}}(i)$

\begin{tabular}{l|c|l}
\hline \hline Types & $p$ & Label series $\left\{l_{t_{1}}(i), \ldots, l_{t_{N}}(i)\right\}$ \\
\hline Unchanged & 1 & $\{1,1, \ldots\}$ \\
Step & 2 & $\{1,1, \ldots, 2,2, \ldots\}$ \\
Impulse & 2 & $\{1,1, \ldots, 2,2, \ldots, 1,1, \ldots\}$ \\
Cycle & 2 & $\{1,1, \ldots, 2,2, \ldots 1,1, \ldots 2,2, \ldots\}$ \\
Complex & $\geqslant 3$ & $\{1,1, \ldots, 2,2 \ldots, 3,3 \ldots 4,4 \ldots\}$ \\
\hline \hline
\end{tabular}

Table 2: The identifications of different types of change.

\section{Experiments}

The proposed methods are evaluated on both synthetic images and real multi-temporal SAR images.

\subsection{Experiments of Change Detection}

\subsubsection{Data Set}

Synthetic images: Fig.6.a shows the noisy synthetic images $y_{t}$ and $y_{t^{\prime}}$ corrupted by single-look multiplicative speckle noise respectively and the ground truth of changes between them. The four squares are $32 \times 32$ pixels with true value 128 . The darker frame is 8 pixels width with 32 as true value and the true value of background is 64 . 
Realistic SAR synthetic images: A denoised image of 21 single-look TerraSAR X-band images in Paris (France) sensed in 2011 is considered as the noise-free image (multi-temporal denoising approach in section 2), as shown in Fig.6.b. Two single-look noisy images $y_{t}$ and $y_{t^{\prime}}$ are generated with changes added in $y_{t^{\prime}}$. These changed regions are about $15-25$ pixels width and length, for instance a $20 \times 20$ pixels patch of vegetation is replaced by a same size patch of building and so on. The right of Fig.6.b shows the ground truth of changes.

Real SAR images 1: 26 single-look TerraSAR images in Saint-Gervaisles-Bains (France) (13 images are sensed in 2009 and the other 13 images in 2011) are shown in Fig.7.a, identified as Saint-Gervais-les-Bains. Reference Ground truth of changes is labeled manually in the right of Fig.7.a.

Real SAR images 2: Experiment in Fig.7.b uses 21 single-look TerraSAR X-band images identified as Paris in Paris (France) sensed in 2011. We label the ground truth of changes manually, as shown in right of Fig.7.b.

Real SAR images 3: Experiment in Fig.9 uses 24 CARABAS-II magnitude images acquired in Vidsel, Sweden 2002, identified as CARABAS (Sensor Data Management System (SDMS) Public web site, 2008). We only detect the changes between image v02_2_1_1 and image v02_4_1_1, while all the 24 images are used in the multi-temporal denoising process.

Real SAR images 4: Experiment in Fig.10 uses 9 single-look TerraSAR X-band images identified as Sendai in Sendai Harbor (Japan) sensed in 2011. Fig.10.a and b show the images acquired respectively on May 6, 2011 and June 8, 2011. All the 9 images are used in the multi-temporal denoising step.

\subsubsection{Change detection methods}

The proposed change criteria approximate likelihood ratio test $\mathcal{R}^{\text {ALRT }}$ and generalized likelihood ratio test $\mathcal{R}^{\mathrm{GLRT}}$ are compared with some stateof-the-art methods, such as Log-Ratio operator (Rignot and van Zyl, 1993), the generalized likelihood ratio test (GLRT) proposed in (Lombardo and Oliver, 2001), Wilcoxon Test based change criterion (Krylov et al., 2012) and Method for generalIzed Means Ordered Series Analysis (Quin et al., 2013), summarized in Tab.3.

\subsubsection{Results}

The change detection results are assessed by the True-Positive versus False-Positive curves using the reference map of changes as shown in Fig.6.c and Fig.7.c. The proposed methods $\mathcal{R}^{\mathrm{ALRT}}$ and $\mathcal{R}^{\mathrm{GLRT}}$ can generally obtain 


\begin{tabular}{|c|c|c|}
\hline Name & Reference & Description \\
\hline Log-Ratio & Rignot and van Zyl (1993) & Log-Ratio operator \\
\hline GLRT & $\begin{array}{l}\text { Lombardo and Oliver } \\
(2001)\end{array}$ & Generalized likelihood ratio test \\
\hline Wilcoxon & Krylov et al. (2012) & $\begin{array}{l}\text { Change detection using a Wilcoxon } \\
\text { Test }\end{array}$ \\
\hline MIMOSA & Quin et al. (2013) & $\begin{array}{l}\text { Method for generalIzed Means Or- } \\
\text { dered Series Analysis }\end{array}$ \\
\hline $\mathcal{R}^{\mathrm{ALRT}}$ & - & $\begin{array}{l}\text { The proposed approximate likeli- } \\
\text { hood ratio test }\end{array}$ \\
\hline $\mathcal{R}^{\mathrm{GLRT}}$ & - & $\begin{array}{l}\text { The proposed generalized likelihood } \\
\text { ratio test }\end{array}$ \\
\hline
\end{tabular}

Table 3: The change detection methods used in the comparison experiments.

higher receiver operating characteristic (ROC) curves than others. The experiments on CARABAS and Sendai data in Fig.9 and 10 show that the proposed GLRT change detection has comparable performance with MIMOSA (Quin et al., 2013). $\mathcal{R}^{\mathrm{ALRT}}$ and $\mathcal{R}^{\mathrm{GLRT}}$ outperform other change criteria, but the latter is more reliable than the former (the ROC curves of $\mathcal{R}^{\mathrm{GLRT}}$ are

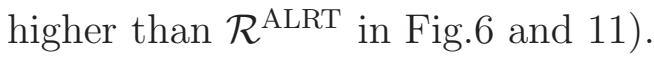

In Saint-Gervais-les-Bains data set, there are only 1817 changed pixels according to the reference map. While, the synthetic data set and the Paris data set have 8116 and 28941 changed pixels respectively (according to the reference map). Too less changed pixels cause the 'noise' in the ROC curves (and all the change detection methods on the Saint-Gervais-les-Bains data set have this 'noise' phenomenon).

\subsection{Experiments of Change Classification}

\subsubsection{Test on realistic $S A R$ synthetic images}

This experiment uses one denoised image of 21 single-look TerraSAR Xband images of Paris (France) sensed in 2011 as the noise-free image (multitemporal denoising approach in section 2), as shown in Fig.6.a. 6 single-look images are generated with different changes added in them. As shown in Fig.6.c, different kinds of changes have been introduced, such as step change (in red), impulse change (in green) and cycle change (in blue). Fig.6.d shows the change classification result by $\mathcal{R}^{\mathrm{GLRT}}$ with confusion matrix shown in Tab.4. 

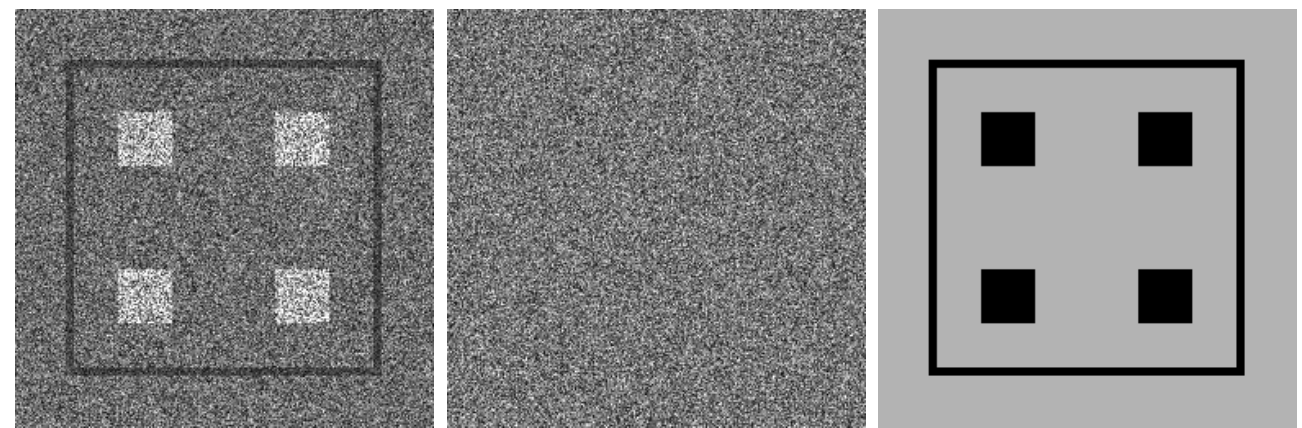

(a) Synthetic images. From left to right: synthetic image $y_{t}$, Synthetic image $y_{t^{\prime}}$ and the reference map of changes.
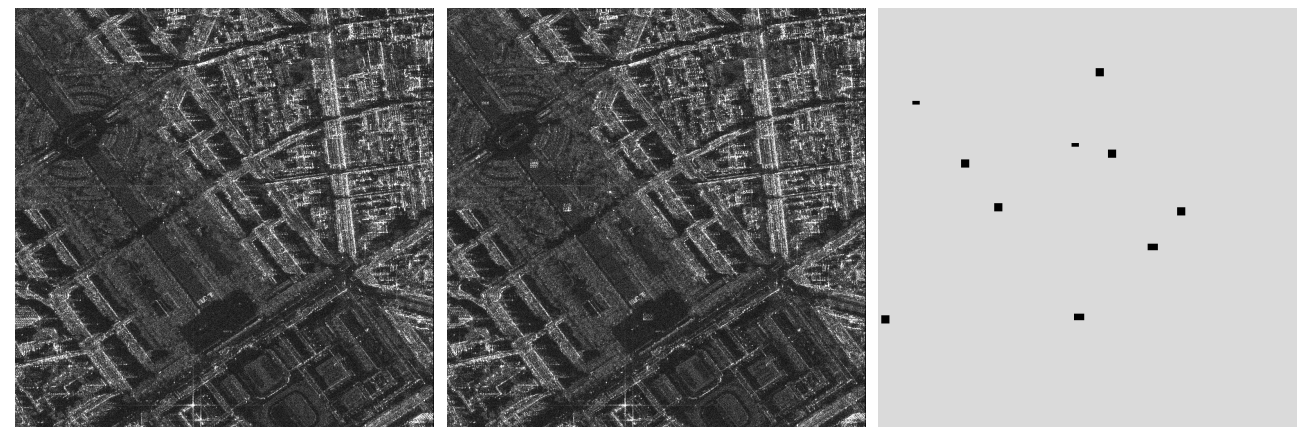

(b) Realistic SAR synthetic images. From left to right: synthetic image $y_{t}$, Synthetic image $y_{t^{\prime}}$ and the reference map of changes.
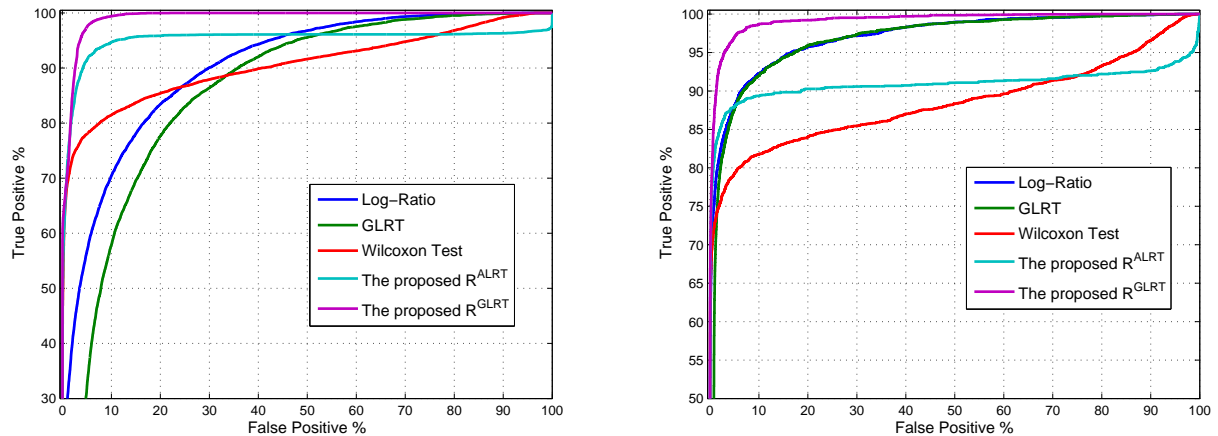

(c) False positive alarm vs true positive curves of synthetic images and realistic SAR synthetic images

Figure 6: Change detection results for synthetic SAR images. 

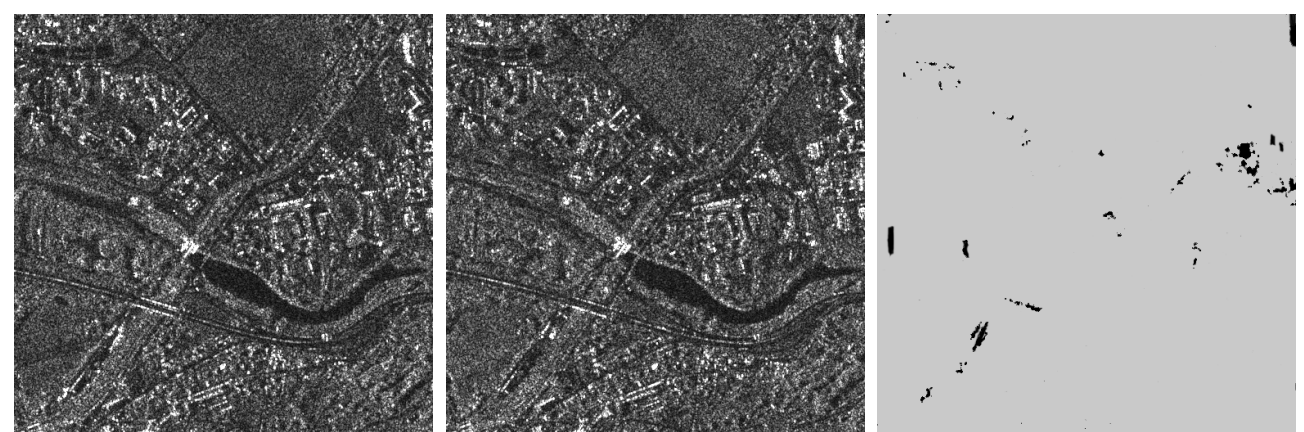

(a) Saint-Gervais-les-Bains data set. From left to right: noisy image $y_{t_{1}}$, noisy image $y_{t_{2} 6}$ and the reference map of changes.
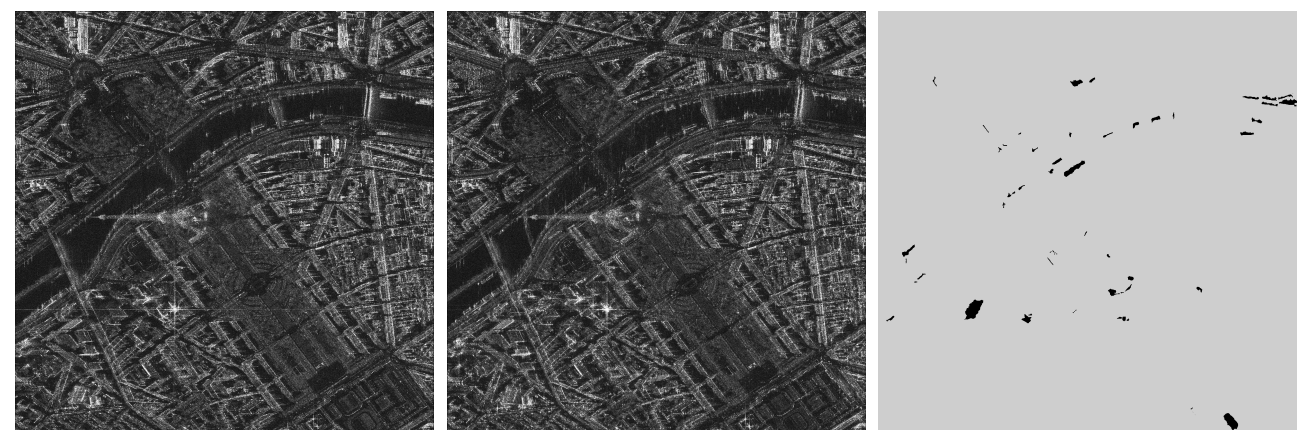

(b) Paris data set. From left to right: noisy image $y_{t_{1}}$, noisy image $y_{t_{2} 6}$ and the reference map of changes.
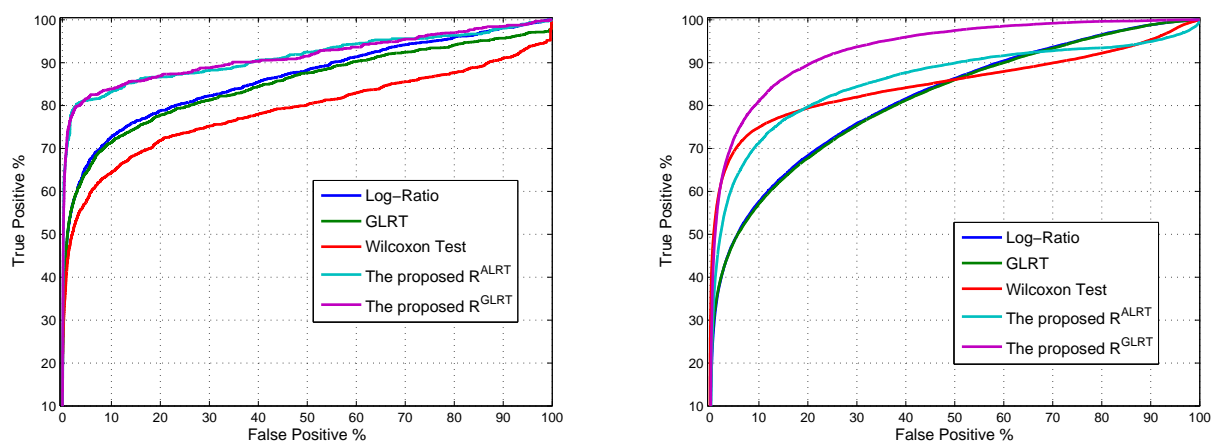

(c) False positive alarm vs true positive curves of Saint-Gervais-les-Bains and Paris data set.

Figure 7: Change detection results for real SAR images Saint-Gervais-les-Bains and Paris data set. 


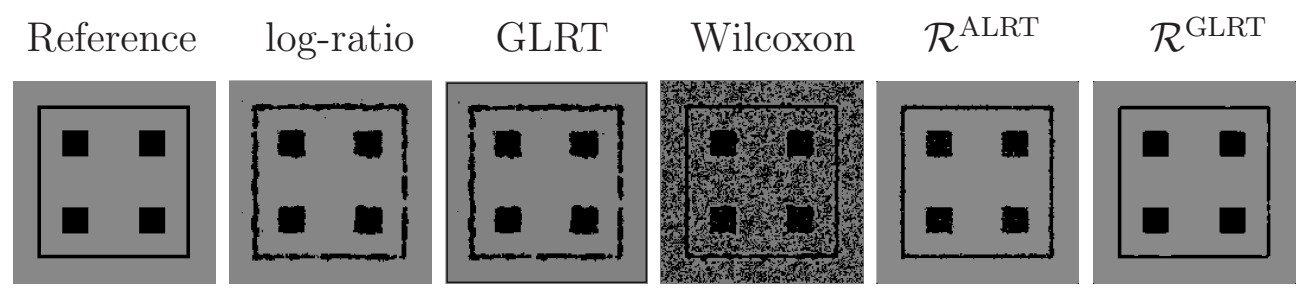

(a) Change detection results of Fig.6.a with True Positive rate 95\%.
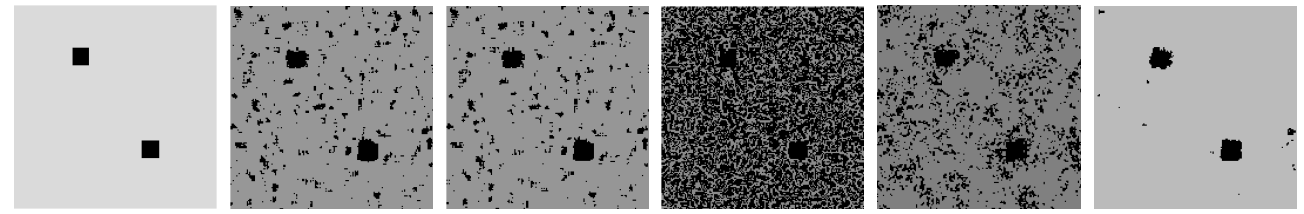

(b) Change detection results of Fig.6.b with True Positive rate 90\%.
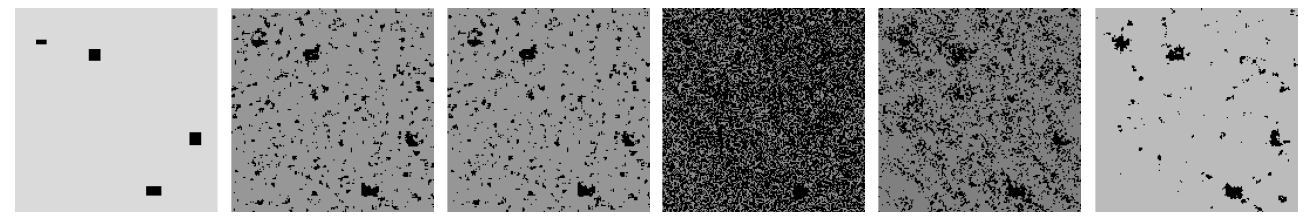

(c) Change detection results of Fig.6.b with True Positive rate 90\%.
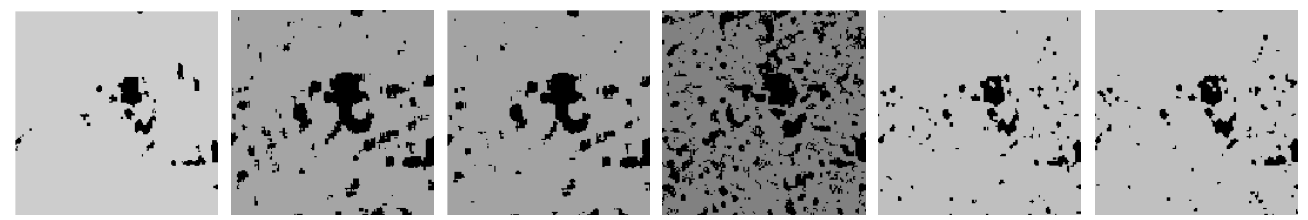

(d) Change detection results of Saint-Gervais-les-Bains data set with True Positive rate $80 \%$.
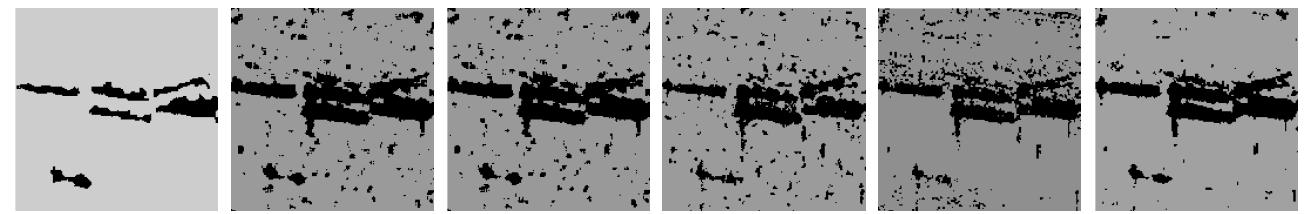

(e) Change detection results of Paris data set with True Positive rate $75 \%$.
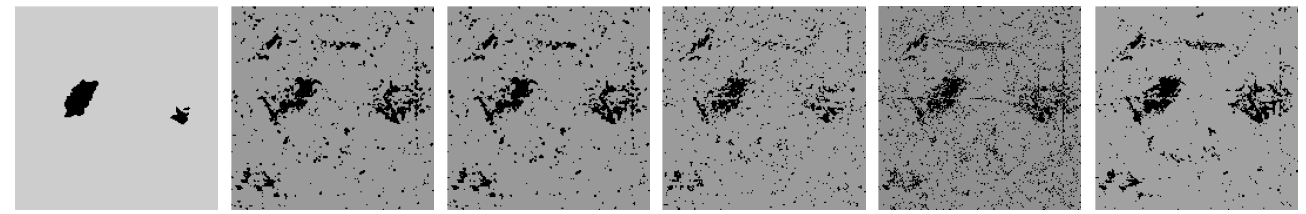

(f) Change detection results of Paris data set with True Positive rate $75 \%$.

Figure 8: Change detection results. 


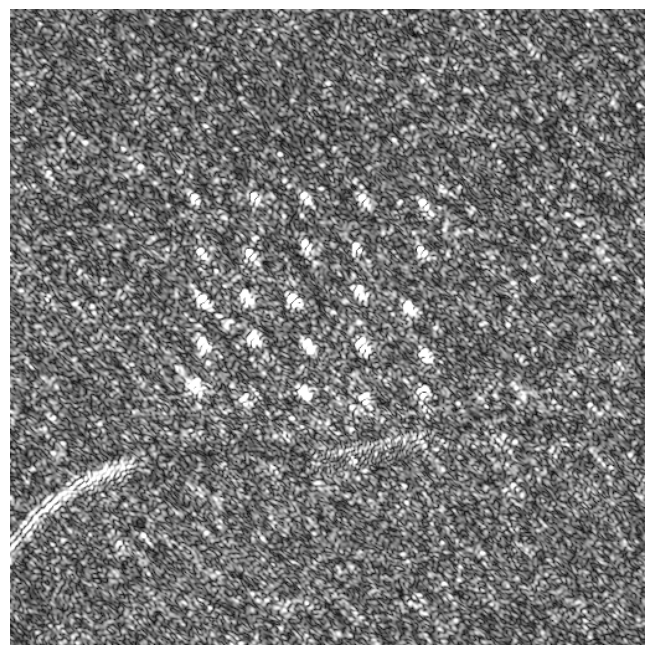

(a) Image v02_2_1_1 in $C A R A B A S$.

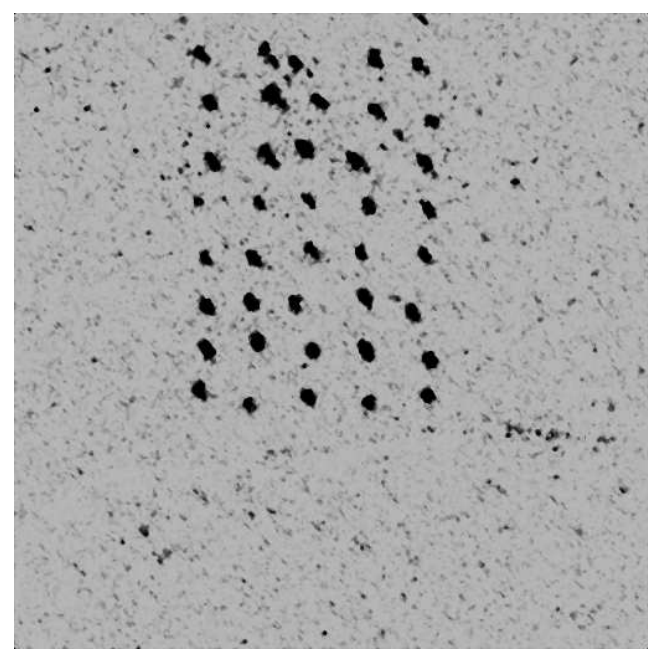

(c) The proposed change criterion $\mathcal{R}^{\text {GLRT }}$. (d) Results using a threshold $\tau$ with $\alpha=$ $0.1 \%$.

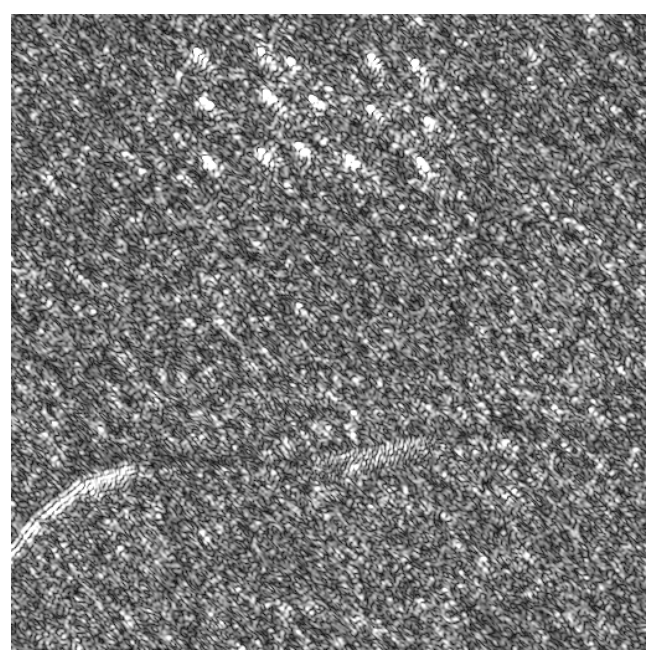

(b) Image v02_4_1_1in $C A R A B A S$.

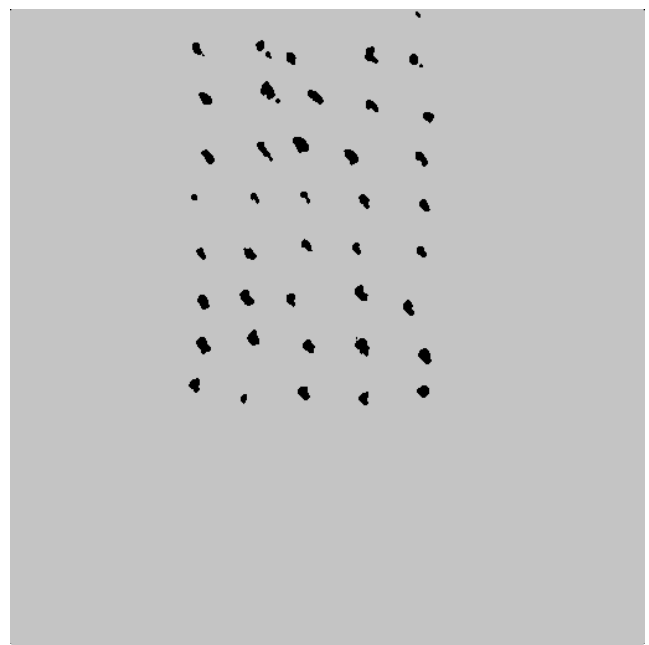

.

Figure 9: Change detection results of real SAR images $C A R A B A S$ (Sensor Data Management System (SDMS) Public web site, 2008). 


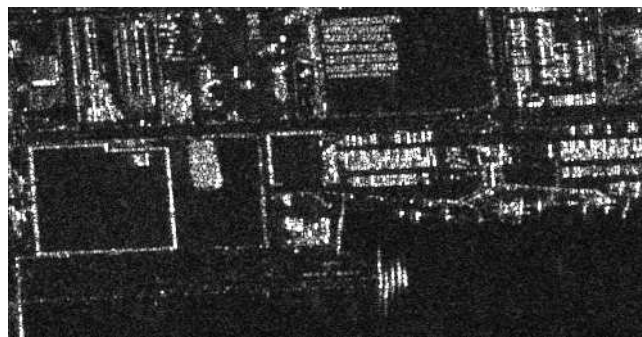

(a) Image on 06/05/2011 in Sendai data.

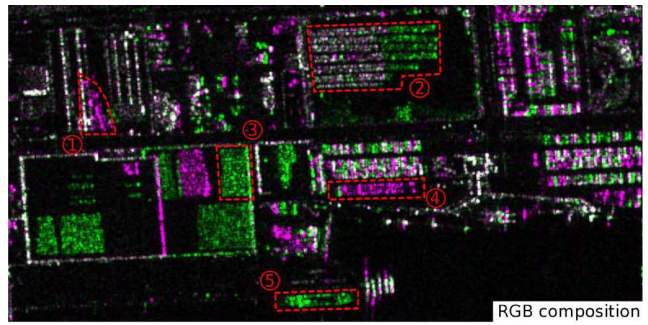

(c) The RGB composition between a and b by MIMOSA (Quin et al., 2013).

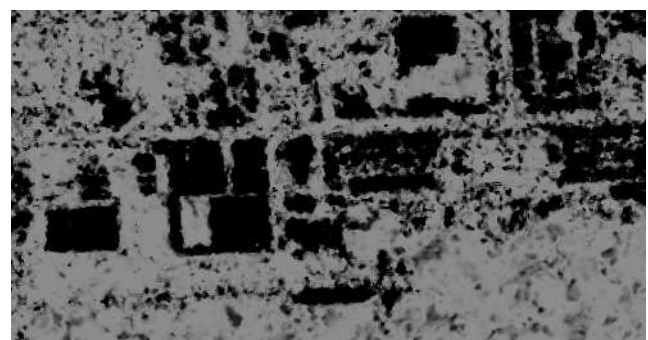

(e) The proposed change criterion $\mathcal{R}^{\mathrm{GLRT}}$

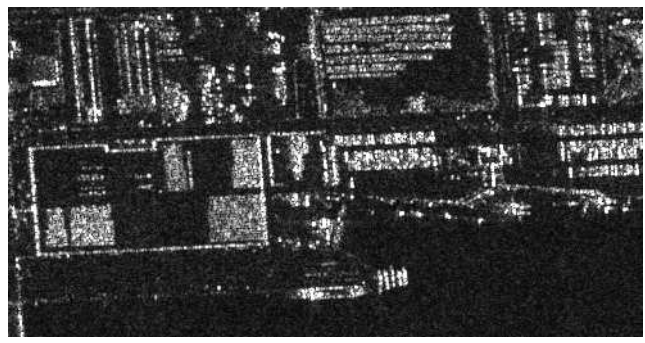

(b) Image on 08/06/2011 in Sendai data.

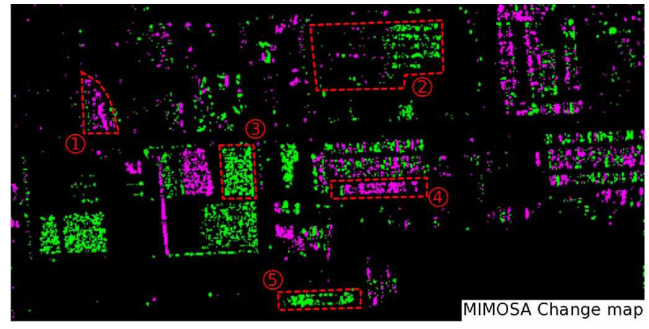

(d) The change detection results by MIMOSA (a prior FAR is 1\%) (Quin et al., 2013).

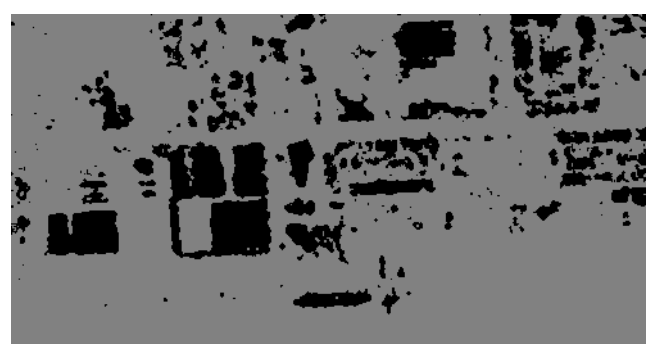

(f) Results using a threshold $\tau$ with $\alpha=$ $0.1 \%$.

Figure 10: Change detection results of real SAR images Sendai. 


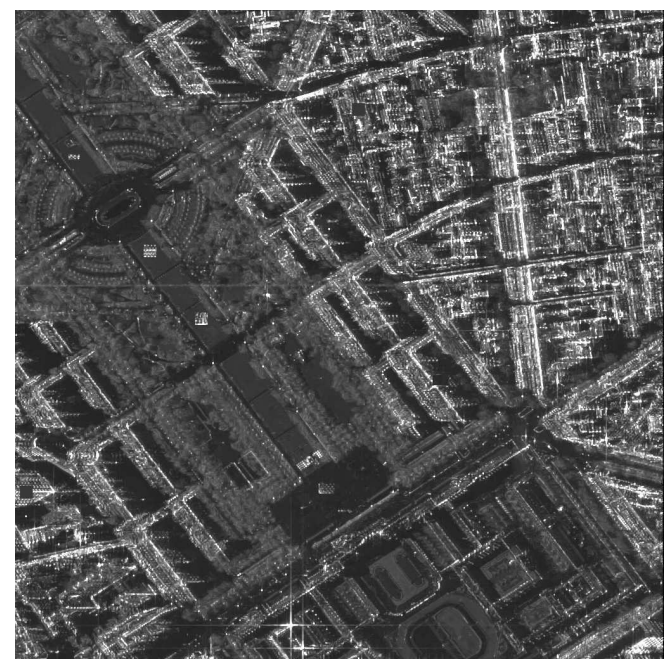

(a) "Noise-free" image with synthetic changes (only 1 image has been shown).

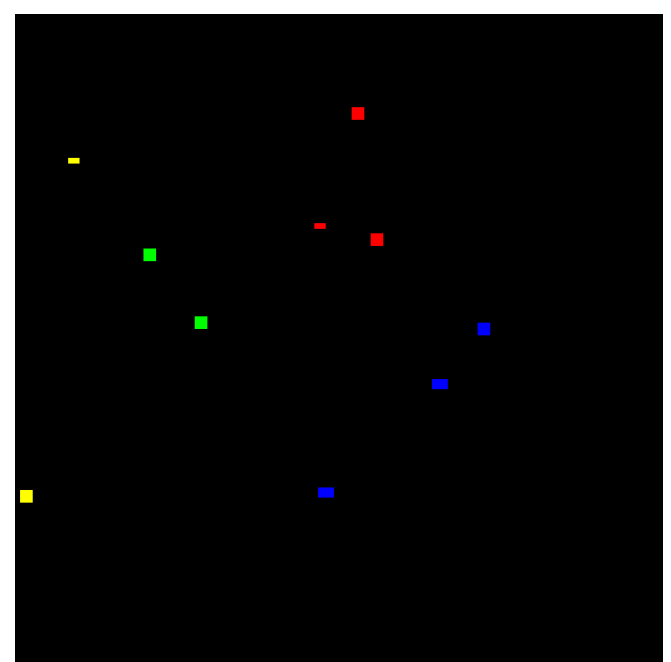

(b) Ground-truth map of changes

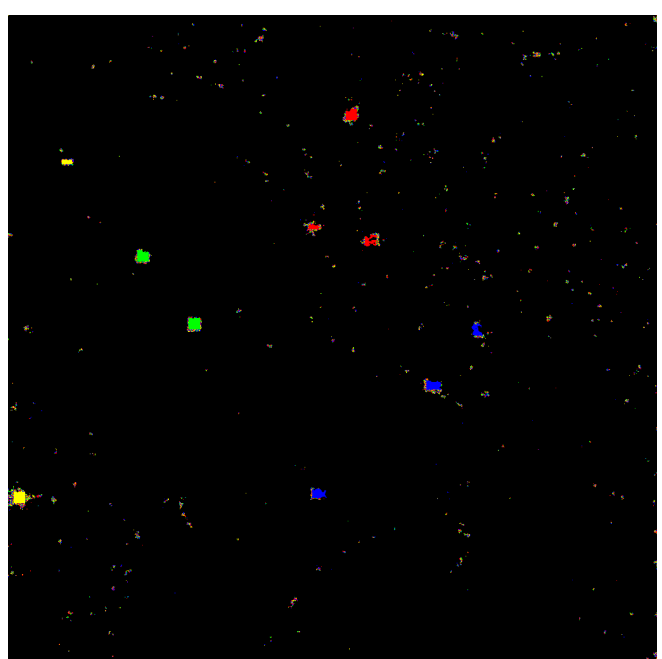

(c) Change classification results by $\mathcal{R}^{\mathrm{GLRT}}$

Figure 11: Change classification of synthetic SAR images (6 single-look SAR images). (a) "noise-free" image with synthetic changes (only 1 image has been shown); (b) Groundtruth map of changes; (c) Change classification results by $\mathcal{R}^{\text {GLRT }}$ (black: no change, red: step change, green: impulse change, blue: cycle change and yellow: complex change. 


\begin{tabular}{|c|c|c|c|c|c|c|}
\hline \multirow{2}{*}{\multicolumn{2}{|c|}{$\begin{array}{c}\text { Confusion } \\
\text { Matrix (\%) }\end{array}$}} & \multicolumn{5}{|c|}{ Classification results } \\
\hline & & Unch. & Step & Impl. & Cyc. & Comp. \\
\hline \multirow{5}{*}{ 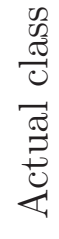 } & Unch & 99.42 & 0.17 & 0.12 & 0.17 & 0.11 \\
\hline & Step & 11.52 & 78.71 & 3.12 & 4.05 & 2.60 \\
\hline & Impl. & 6.25 & 4.50 & 80.25 & 5.63 & 3.38 \\
\hline & Cyc. & 17.50 & 2.92 & 1.58 & 75.58 & 2.42 \\
\hline & Comp. & 4.27 & 5.52 & 2.85 & 6.23 & 81.14 \\
\hline
\end{tabular}

Table 4: Confusion matrix of change classification results. Unch.: unchanged, Step: step change, Impl.:impulse change, Cyc.: cycle change and Comp.: complex change.

\subsubsection{Test on real $S A R$ images}

We have 21 single-look TerraSAR X-band images identified as Paris in Paris (France) sensed in 2011 and 6 single-look TerraSAR X-band images identified as San-Francisco sensed in San-Francisco, U.S.A. 2007 and 2011. These images have been accurately registered using the sensor parameters. Fig.12.a and b only show the first noisy image and its denoising result. Fig.12.c shows the results of the change classification approach by $\mathcal{R}^{\text {GLRT }}$, in which red regions denote step changes, green are impulse changes and blue are cycle changes. We can observe that many boats in river have been classified as impulse change. Fig.13 gives an illustration of examples of step change, impulse change; cycle change and complex change with the corresponding optical images (C) Google (but the dates of the optical images are not exactly the same as the SAR images). We can see that the step changes probably corresponds to the presence of some facilities in the stadium, impulse and complex changes corresponds to boats that are moored at piers, while cycle changes might correspond to the river bank.

\section{Conclusion}

In this work, a global framework NORmalized Cut on chAnge criterion MAtrix (NORCAMA) for change classification of multi-temporal SAR time series has been presented. To reduce the effect of speckle, a multi-temporal denoising approach is applied in the pre-processing step. Using both noisy data and denoised data, the approximate likelihood ratio and the generalized likelihood ratio are computed as change criteria. The change classification is performed by clustering on change criterion matrix and classifying of label transformation. Different types of change, like step changes, impulse changes 

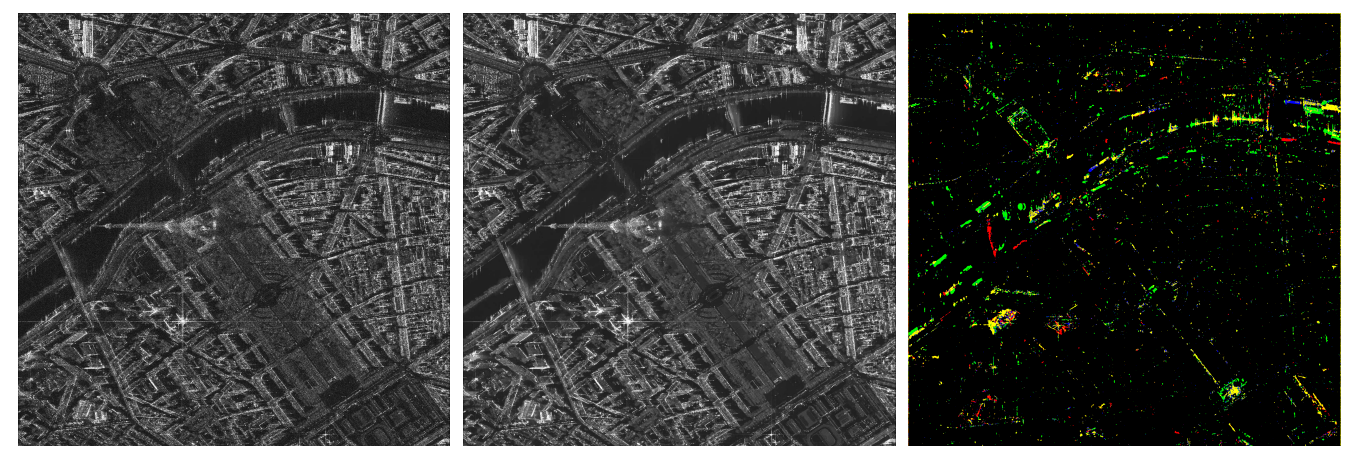

(a) Paris, 21 single-look TerraSAR-X images
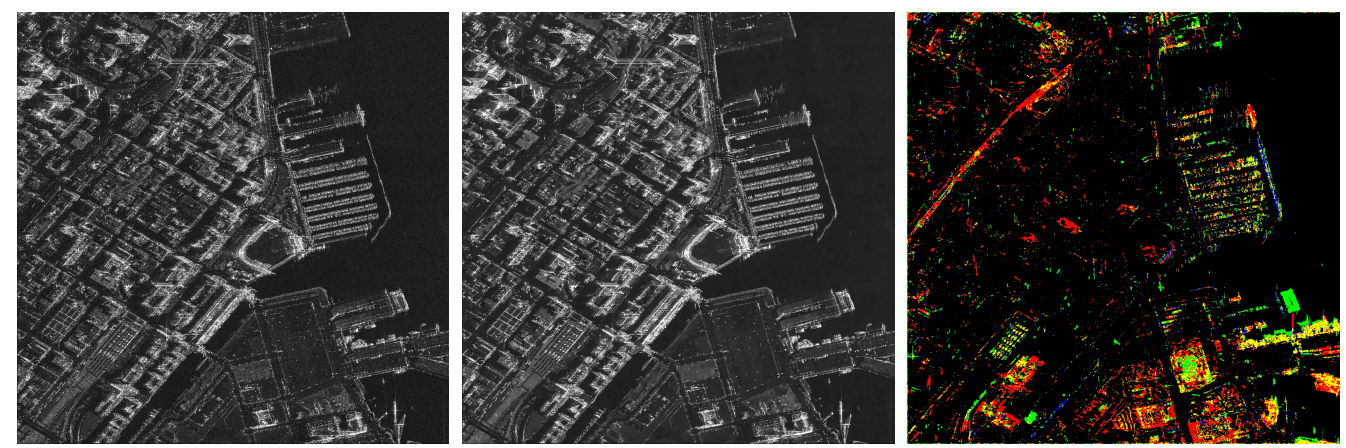

(b) San-Francisco, 6 single-look TerraSAR-X images

Figure 12: Change classification on Real SAR images. From top to bottom: Paris, 21 single-look TerraSAR-X images and San-Francisco, 6 single-look TerraSAR-X images. From left to right: noisy image $y_{t_{1}}$, multi-temporal denoising result of $y_{t_{1}}$ and change classification results by criterion $\mathcal{R}^{\mathrm{GLRT}}$. (black: no change, red: step change, green: impulse change, blue: cycle change yellow: complex change.) 

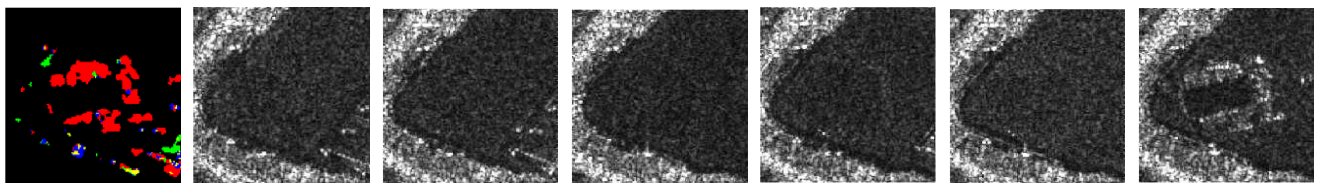

(a) The AT\&T park in San-Francisco. Example of step change in San-Francisco. From left to right, the change classification results, the noisy image from date $t_{1}$ to $t_{6}$.
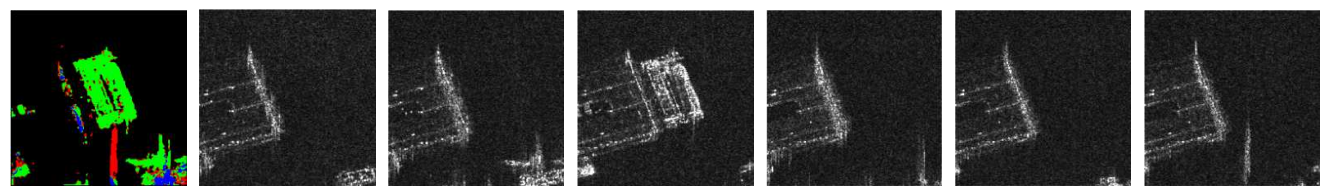

(b) The Pier 48 in San-Francisco. Example of impulse change in San-Francisco. From left to right, the change classification results, the noisy image from date $t_{1}$ to $t_{6}$.
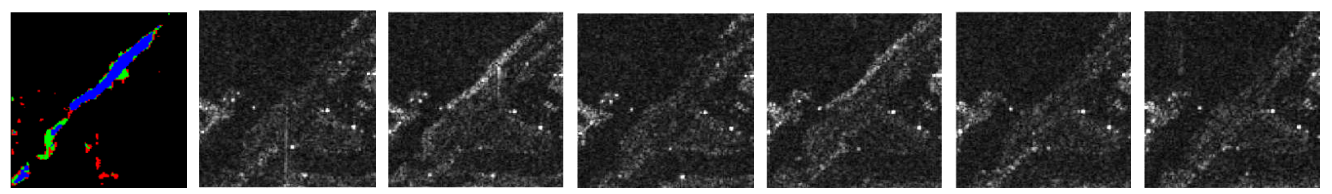

(c) The bank of the river in the Mission Creek Garden. Example of cycle change in San-Francisco. From left to right, the change classification results, the noisy image from date $t_{1}$ to $t_{6}$.
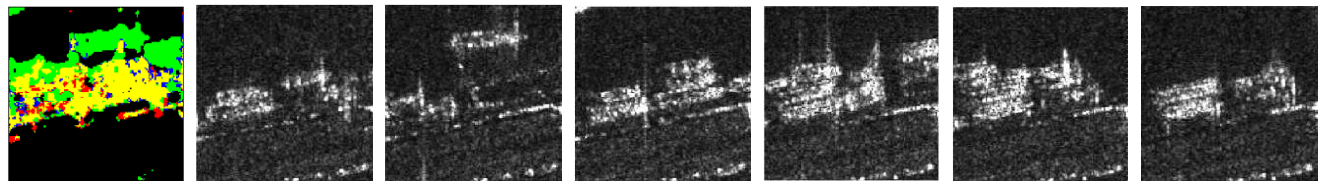

(d) The Pier 50 in San-Francisco. Example of complex change in San-Francisco. From left to right, the change classification results, the noisy image from date $t_{1}$ to $t_{6}$.

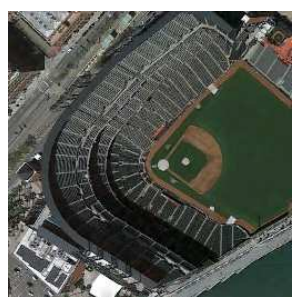

(e)

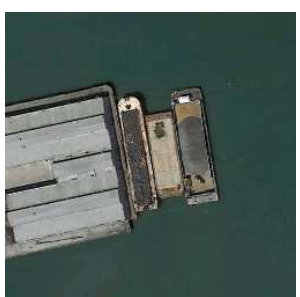

(f)

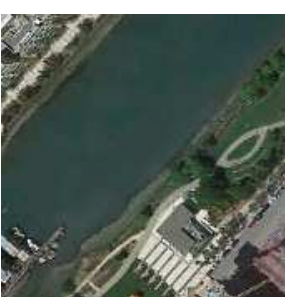

$(\mathrm{g})$

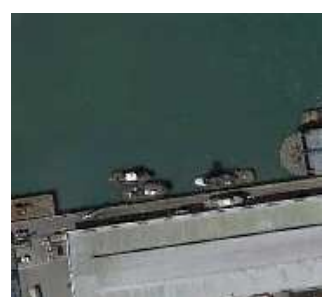

(h)

Figure 13: Details of change classification results of San-Francisco data set. (a-d) from top to bottom: from left to right: the AT\&T park, the Pier 48, the bank of the river in the Mission Creek Garden and the Pier 50. They are step change, impulse change; cycle change and complex change. (black: no change, red: step change, green: impulse change, blue: cycle change and yellow: complex change). (e-h) the optical images (imagery data 2014.02.24) corresponding to images (a-d). 
and cycle changes, have been defined by the proposed method, which can be used for multi-temporal SAR image interpretation. Our proposed framework is flexible enough to consider alternative methods during the three steps, for instance other denoising approaches for the pre-processing step and other change criteria for the change detection step.

The future work will be focused in introducing spatial information into change analysis such as combining types of change and shapes to identify objects and developing a temporal-spatial SAR image analysis framework. The spatial information should allow us to extend this approach to multiincidence images. Extension of our method for the classification of complex changes with more than three clusters constitutes another perspective. This would require a more detailed analysis for the complex change and more information on the application context.

\section{Acknowledgment}

The authors would like to thank Jean-Marie Nicolas for his help on image registration, the IGARSS Data Fusion Contest for providing the multitemporal SAR images and the Department of Radar Systems, Swedish Defense Research Agency (FOI), for the CARABAS-II data distribution. They also wish to acknowledge the German Aerospace Agency (DLR) for the TerraSAR-X images (project MTH0232 and LAN1746).

\section{References}

Atto, A.M., Trouvé, E., Berthoumieu, Y., Mercier, G., 2013. Multidate Divergence Matrices for the Analysis of SAR Image Time Series. IEEE Transactions on Geoscience and Remote Sensing 51, 1922-1938.

Bazi, Y., Bruzzone, L., Melgani, F., 2005. An unsupervised approach based on the generalized Gaussian model to automatic change detection in multitemporal SAR images. IEEE Transactions on Geoscience and Remote Sensing 43, 874-887.

Bovolo, F., Bruzzone, L., 2005. A detail-preserving scale-driven approach to change detection in multitemporal SAR images. IEEE Transactions on Geoscience and Remote Sensing 43, 2963-2972. 
Brunner, D., Lemoine, G., Bruzzone, L., 2010. Earthquake damage assessment of buildings using VHR optical and SAR imagery. IEEE Transactions on Geoscience and Remote Sensing 48, 2403-2420.

Bruzzone, L., Marconcini, M., Wegmuller, U., Wiesmann, A., 2004. An advanced system for the automatic classification of multitemporal SAR images. IEEE Transactions on Geoscience and Remote Sensing 42, 13211334.

Bruzzone, L., Prieto, D., 2000. Automatic analysis of the difference image for unsupervised change detection. IEEE Transactions on Geoscience and Remote Sensing 38, 1171-1182.

Bruzzone, L., Serpico, S., 1997. An iterative technique for the detection of land-cover transitions in multitemporal remote-sensing images. IEEE Transactions on Geoscience and Remote Sensing 35, 858-867.

Buades, A., Coll, B., Morel, J., 2005. A non-local algorithm for image denoising, in: IEEE Computer Society Conference on Computer Vision and Pattern Recognition, IEEE. pp. 60-65.

De Jong, R., de Bruin, S., de Wit, A., Schaepman, M., Dent, D., 2011. Analysis of monotonic greening and browning trends from global NDVI time-series. Remote Sensing of Environment 115, 692-702.

Deledalle, C., Denis, L., Tupin, F., 2009. Iterative weighted maximum likelihood denoising with probabilistic patch-based weights. IEEE Transactions on Image Processing 18, 2661-2672.

Deng, J., Wang, K., Deng, Y., Qi, G., 2008. PCA-based land-use change detection and analysis using multitemporal and multisensor satellite data. International Journal of Remote Sensing 29, 4823-4838.

Fung, T., 1990. An assessment of TM imagery for land-cover change detection. IEEE Transactions on Geoscience and Remote Sensing 28, 681-684.

Fung, T., LeDrew, E., 1987. Application of principal components analysis to change detection. Photogrammetric Engineering and Remote Sensing 53, 1649-1658. 
Goodman, J., 1976. Some fundamental properties of speckle. JOSA 66, 1145-1150.

Huang, C., Wylie, B., Yang, L., Homer, C., Zylstra, G., 2002. Derivation of a tasselled cap transformation based on Landsat 7 at-satellite reflectance. International Journal of Remote Sensing 23, 1741-1748.

Julea, A., Méger, N., Bolon, P., Rigotti, C., Doin, M., Lasserre, C., Trouvé, E., Lazarescu, V., 2011. Unsupervised spatiotemporal mining of satellite image time series using grouped frequent sequential patterns. IEEE Transactions on Geoscience and Remote Sensing 49, 1417-1430.

Julea, A., Méger, N., Rigotti, C., Trouvé, E., Jolivet, R., Bolon, P., et al., 2012. Efficient Spatio-temporal Mining of Satellite Image Time Series for Agricultural Monitoring. IEEE Transactions on Machine Learning and Data Mining 5, 23-44.

Kay, S., 1998. Fundamentals of statistical signal processing, Volume II: Detection theory. Upper Saddle River (New Jersey) 7.

Kervrann, C., Boulanger, J., 2006. Optimal spatial adaptation for patchbased image denoising. IEEE Transactions on Image Processing 15, 28662878 .

Krylov, V.A., Moser, G., Voisin, A., Serpico, S.B., Zerubia, J., 2012. Change detection with synthetic aperture radar images by Wilcoxon statistic likelihood ratio test, in: IEEE International Conference on Image Processing, IEEE. pp. 2093-2096.

Lombardo, P., Oliver, C., 2001. Maximum likelihood approach to the detection of changes between multitemporal SAR images, in: IEE Proceedings on Radar, Sonar and Navigation, IET. pp. 200-210.

Lu, P., Stumpf, A., Kerle, N., Casagli, N., 2011. Object-oriented change detection for landslide rapid mapping. IEEE Geoscience and Remote Sensing Letters 8, 701-705.

Marchesi, S., Bruzzone, L., 2009. ICA and kernel ICA for change detection in multispectral remote sensing images, in: IEEE International Conference on Geoscience and Remote Sensing Symposium, IEEE. pp. II-980. 
Martínez, B., Gilabert, M., 2009. Vegetation dynamics from NDVI time series analysis using the wavelet transform. Remote Sensing of Environment 113, $1823-1842$.

Preiss, M., Stacy, N., 2006. Coherent change detection: theoretical description and experimental results. Technical Report. DTIC Document.

Quin, G., Pinel-Puyssegur, B., Nicolas, J.M., Loreaux, P., 2013. MIMOSA: An Automatic Change Detection Method for SAR Time Series. IEEE Transactions on Geoscience and Remote Sensing 52, 5349 - 5363.

Radke, R.J., Andra, S., Al-Kofahi, O., Roysam, B., 2005. Image change detection algorithms: a systematic survey. IEEE Transactions on Image Processing 14, 294-307.

Rignot, E., van Zyl, J., 1993. Change detection techniques for ERS-1 SAR data. IEEE Transactions on Geoscience and Remote Sensing 31, 896-906.

Sensor Data Management System (SDMS) Public web site, 2008. VHF Change Detection Problem Set. https://www. sdms . afrl . af .mil/index. php?collection=vhf_change_detection. [Online].

Shi, J., Malik, J., 2000. Normalized cuts and image segmentation. IEEE Transactions on Pattern Analysis and Machine Intelligence 22, 888-905.

Singh, A., 1989. Digital change detection techniques using remotely-sensed data. International journal of remote sensing 10, 989-1003.

Su, X., Deledalle, C.A., Tupin, F., Sun, H., 2014. Two-Step Multitemporal Nonlocal Means for Synthetic Aperture Radar Images. IEEE Transactions on Geoscience and Remote Sensing 52, 6181-6196.

Taubenböck, H., Esch, T., Felbier, A., Wiesner, M., Roth, A., Dech, S., 2012. Monitoring urbanization in mega cities from space. Remote sensing of Environment 117, 162-176.

Verbesselt, J., Hyndman, R., Newnham, G., Culvenor, D., 2010a. Detecting trend and seasonal changes in satellite image time series. Remote sensing of Environment 114, 106-115. 
Verbesselt, J., Hyndman, R., Zeileis, A., Culvenor, D., 2010b. Phenological change detection while accounting for abrupt and gradual trends in satellite image time series. Remote Sensing of Environment 114, 2970-2980.

Von Luxburg, U., 2007. A tutorial on spectral clustering. Statistics and computing 17, 395-416.

Yin, J., Yin, Z., Zhong, H., Xu, S., Hu, X., Wang, J., Wu, J., 2011. Monitoring urban expansion and land use/land cover changes of Shanghai metropolitan area during the transitional economy (1979-2009) in China. Environmental monitoring and assessment 177, 609-621. 